\title{
Experiments on the Richtmyer-Meshkov instability: Wall effects and wave phenomena
}

\author{
M. Brouillette ${ }^{\text {a) }}$ \\ Département de génie mécanique, Université de Sherbrooke, Sherbrooke, Québec JIK 2R1, Canada \\ R. Bonazza \\ Department of Nuclear Engineering, University of Wisconsin, Madison, Wisconsin 53704
}

(Received 17 May 1995; accepted 22 January 1999)

\begin{abstract}
Experiments examining the interaction of shock waves with an interface separating two gases of different densities are reported. Flow visualization by the schlieren method and x-ray densitometry reveals that important secondary effects are introduced by the experimental apparatus, especially at the walls of the shock tube from shock wave/boundary layer interaction below, above, and at the interface itself. These effects can impair the observation of the primary phenomenon under study and can lead to the overall deformation of the interface. In particular, the thickness of the viscous boundary layer at the interface is computed using a familiar shock tube turbulent boundary layer model and the occurrence of bifurcation of reflected waves below and above the interface is successfully predicted based on classical bifurcation arguments. The formation of wall vortical structures at the interface is explained in terms of baroclinic vorticity deposition resulting from the interaction of reflected waves with the interface distorted by the boundary layer. This mechanism of wall vortex formation can also explain observed test gas contamination in reflected shock tunnels when shock wave bifurcation is absent. In general, it is found that most of the side effects of the experimental investigation of the Richtmyer-Meshkov instability can be alleviated by performing experiments in large test sections near atmospheric initial pressure. (c) 1999 American Institute of Physics. [S1070-6631(99)01505-6]
\end{abstract}

\section{INTRODUCTION}

The impulsive acceleration of an interface separating two fluids of different densities can lead to a wide variety of fluid motions. Any perturbation initially present on the interface will be distorted through baroclinic vorticity generation. At the later stages of development of the interface, the interpenetration of the two fluids can generate even more vorticity through the shearing instability.

This class of flows is often referred to as the shockexcited Rayleigh-Taylor instability, or as the RichtmyerMeshkov instability, for the two scientists who first presented theoretical (Richtmyer ${ }^{1}$ ) and experimental $\left(\right.$ Meshkov $\left.^{2}\right)$ results. The past twenty years have seen a strong research interest in this class of problems, because of potential applications mainly in laser implosion of deuteriumtritium nuclear fusion targets and hypervelocity mixing/ combustion. Giant steps have been made towards the numerical simulation of these flows, validated with laboratory experiments usually performed in shock tubes.

Shock tube experiments on the Richtmyer-Meshkov instability have been performed in many countries. Agreement between the different results has not been good, owing to the large discrepancy in experimental facilities, diagnostic methods, and test conditions. ${ }^{3}$ In fact, the experimental study of interaction of shock waves with an interface between gases of different densities ${ }^{3-5}$ has demonstrated that secondary ef-

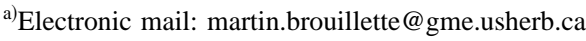

fects, introduced by the apparatus, can impair the development and observation of the primary phenomenon under study.

The present work describes and classifies the secondary effects that can take place during the experimental investigation of the Richtmyer-Meshkov instability: these have to be understood in order to correctly interpret past and present data and also to assist in planning future experiments. This article is therefore intended as a repertory and guide for experimental and numerical investigators in the field. In particular, the influence of these secondary effects on the interpretation of usual experimental results, such as the measurement of interface turbulent thickening and perturbation growth rates, are discussed. These effects all originate from the repeated interaction of shock waves with the boundary layer developing at the interface. In particular, it is found that the propagation of shock waves over the wall boundary layers can lead to wave bifurcation, and that the interaction of these waves with the distorted interface within the boundary layers causes the formation of a wall vortex which can deform the interface by vorticity-induced strain. It is also found that two- (2D) and three-dimensional (3D) wave fronts are generated by disturbances on the interface and on the side and end walls of the shock tube, and by interaction with the boundary layers. After these waves reverberate between the side and end walls of the shock tube and the interface itself, they are responsible for the introduction of small perturba- 
tions on the interface, which can explain the observed thickening of initially smooth thick interfaces.

\section{EXPERIMENTAL FACILITY}

Using schlieren photography and x-ray densitometry, we study the development of the interfacial instability produced by the interaction of shock and expansion waves parallel to an interface separating two gases of different densities. Experiments are performed in the GALCIT vertical shock tube, where a shock wave is launched from the top of the tube toward the interface located near the bottom end wall. The driven section of the shock tube is square throughout its length, with a $114 \mathrm{~mm}$ side, and mates with a test section of the same sectional dimensions; this eliminates the need for the cookie cutter section usually necessary between a round driven section and a square test section. More details about the apparatus, the schlieren flow visualization setup, and the $\mathrm{x}$-ray densitometry system can be obtained from Brouillette ${ }^{6}$ and Bonazza. ${ }^{7}$

The experiments are performed with two different kinds of interfaces: (i) a discontinuous interface formed by a thin $(0.5 \mu \mathrm{m})$ nitrocellulose membrane and (ii) a thick, membrane-free, continuous interface smoothed by molecular diffusion, formed by withdrawing a thin $(1.2 \mathrm{~mm})$ horizontal metal plate initially separating the gases.

In all cases atmospheric air is used above the interface and helium $(\mathrm{He})\left(\right.$ density $0.16 \mathrm{~kg} / \mathrm{m}^{3}$ at $\left.25^{\circ} \mathrm{C}, 1 \mathrm{~atm}\right)$, carbon dioxide $\left(\mathrm{CO}_{2}\right)\left(1.8 \mathrm{~kg} / \mathrm{m}^{3}\right)$, freon-22 (R-22) $\left(3.5 \mathrm{~kg} / \mathrm{m}^{3}\right)$, xenon $(\mathrm{Xe})\left(5.5 \mathrm{~kg} / \mathrm{m}^{3}\right)$, and sulfur hexafluoride $\left(\mathrm{SF}_{6}\right)(6.0$ $\mathrm{kg} / \mathrm{m}^{3}$ ), are used below the interface as test gases.

The interface can be installed within the field of view of the flow visualization windows or at two locations (32 or 65 $\mathrm{cm}$ ) upstream of it. The location of the end wall of the shock tube with respect to the windows can also be moved, allowing us to change the period of the waves reverberating between the interface and the end wall. Although a large number of combinations are possible, two configurations are used: (i) The "short period" experiments are performed with the interface initially in the field of view of the windows, and the end wall of the shock tube is moved just $10 \mathrm{~cm}$ downstream of the interface [Fig. 1(a)]. This setup is used to observe the evolution of the interface at early times after the interaction with the incident shock and also to study the effects of multiple impulsive accelerations caused by reverberations of the primary wave between the interface and the end wall of the shock tube. (ii) In the other configuration, the "long period" experiments, the interface is initially located either 32 or $65 \mathrm{~cm}$ upstream of the flow visualization windows [Fig. 1(b)]. The end of the shock tube is adjusted so that the first reflection from the end wall interacts with the interface as the latter is near the bottom of the window. Thus, a "snapshot" view of the interface a long time after it interacts with the incident shock, as well as of the effects of the first reshock, are obtained.

For the optical flow visualization setup, the sensitivity of the schlieren system is adjusted so that the interface can easily be distinguished from the image of the shock waveboundary layer interaction on the observing window. At the

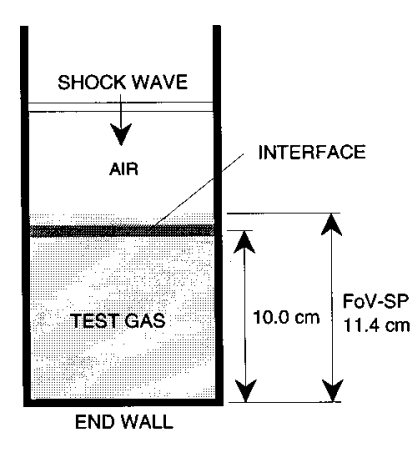

(a)

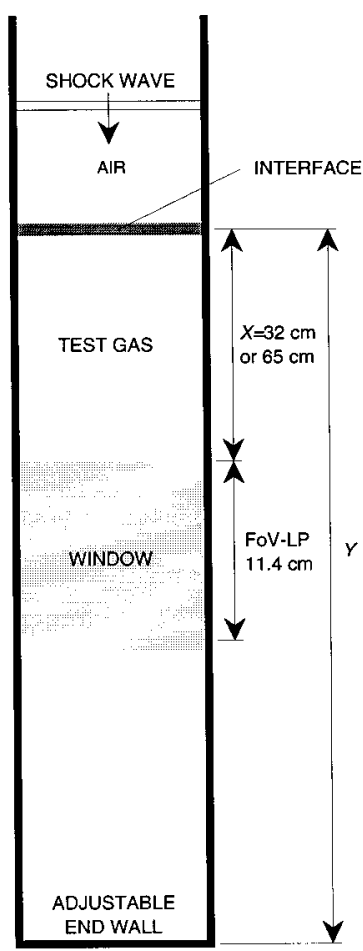

(b)
FIG. 1. Experimental configurations. (a) Short period experiments. The interface is initially placed within the field of view of the windows and the end wall of the shock tube is positioned just $10 \mathrm{~cm}$ downstream of the initial position of the interface. This setup is used to observe the evolution of the interface at early times after the interaction with the incident shock and also to study the effects of multiple impulsive accelerations caused by reverberations of the primary wave between the interface and the end wall of the shock tube. The field of view of the flow visualization system is indicated by "FoV-SP." (b) Long period experiments. The distance $X$ between the initial position of the interface and the top of the window can be set to 32 or $65 \mathrm{~cm}$ and the distance $Y$ between the interface and the end wall is adjusted to observe the interaction of the first reshock with the interface as the latter is near the lower end of the window. The field of view of the flow visualization system is indicated by "FoV-LP."

same time, the sensitivity is kept sufficient to record the random field of acoustic waves associated with the shock waves reverberating between the interface and the end of the tube. For examining the fine details of the interaction between the two gases, high-resolution spark schlieren photography is used, which yields one photograph per experimental run. To measure the time evolution of the interface we use highspeed [35 000-60 000 frames per second (fps)] schlieren motion pictures which yield images of lower resolution. To quantify the density field in the interface region, an x-ray flow visualization system is used, with which a single $50 \mathrm{~ns}$ flash is produced for each experimental run and an image is recorded by an X-ray negative sandwiched between two fluorescent screens. The $\mathrm{x}$-ray negative is subsequently digitized using a charge-coupled device (CCD) camera and the density field information extracted through a custom image-analysis computer routine. ${ }^{7}$

To perform a run, the sliding plate or membrane is fully inserted into the test section, and the test gas is introduced below the interface. A microprocessor-driven control system is used to provide the sequence for retracting the plate (when 

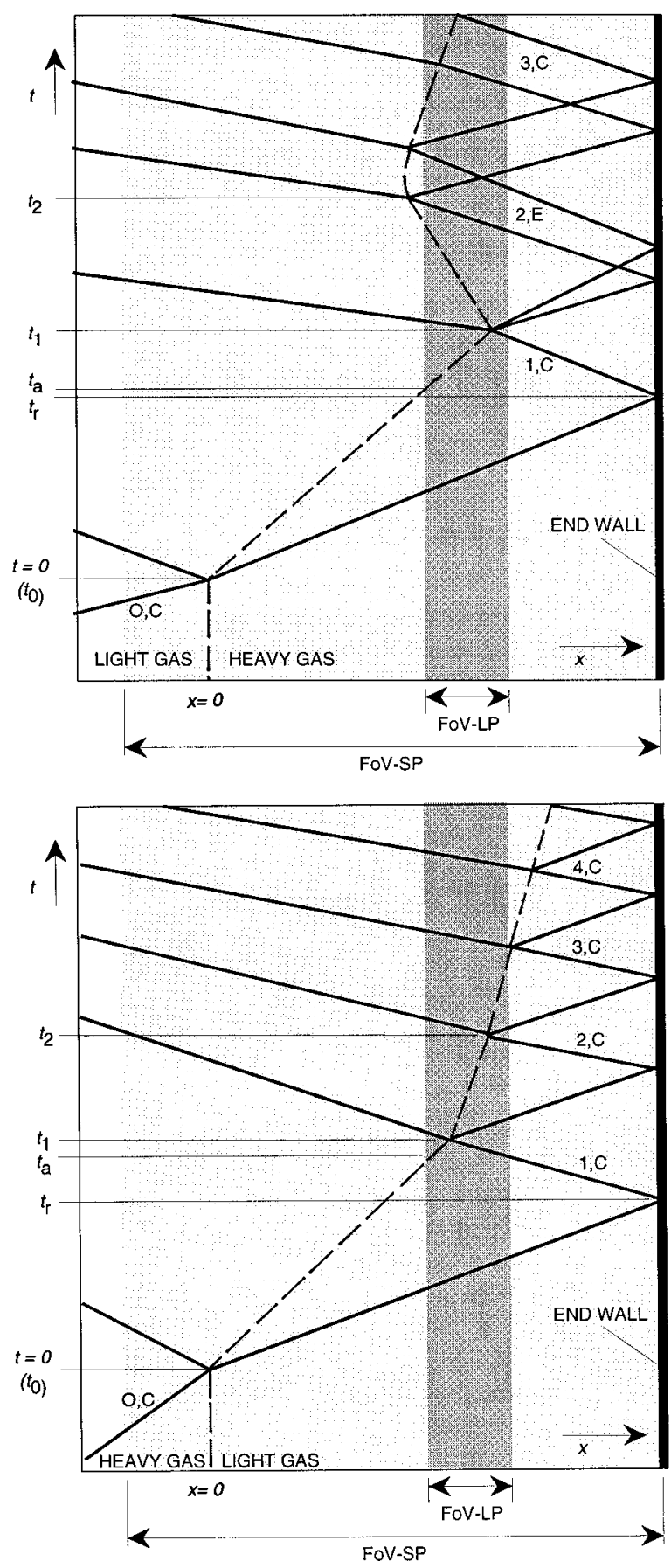

FIG. 2. (a) Generic wave diagram for the interaction of a moderate strength shock wave with a light/heavy interface. (b) Generic wave diagram for the interaction of a moderate strength shock wave with a heavy/light interface. The interface trajectory is indicated by: --- . The wave trajectories are indicated by: - Waves successively interacting with the interface are numbered 0, 1, 2, etc.; $\mathbf{C}$ indicates compression waves, $\mathbf{E}$ expansion waves. The field of view of the flow visualization system in the short period configuration is indicated by FoV-SP (light shading) and by FoV-LP (heavy shading) for experiments in the long period configuration.

applicable), firing the shock wave and triggering the flow visualization equipment. All the experiments are performed at atmospheric initial conditions in the test section, and the Mach number of the incident shock wave was varied from $M_{s}=1.10$ to 1.70 .

Figure 2 shows generic wave diagrams for the interac- tion of a shock of moderate strength with an interface separating a light and a heavy gas [Fig. 2(a)] and an interface between a heavy and a light gas [Fig. 2(b)]. The incident shock of strength $M_{s}$ interacts with the interface at position $x=0$ at time $t=0$. For the light/heavy interface [Fig. 2(a)], the refraction of the incident shock at the interface transmits a shock wave into the heavy gas (shock strength $M_{t}$ ) and a shock is also reflected back into the light gas. The interface is impulsively set in motion to a velocity $[u]_{0}$. The transmitted wave subsequently reflects from the end wall of the shock tube at $t=t_{r}$ and interacts with the interface at $t=t_{1}$. This first reflected wave, referred to as the first reshock, is labeled wave 1 , and imparts to the interface a change in velocity $[u]_{1}$ after transmitting a shock wave into air above the interface and reflecting expansion waves in the test gas below. Further reverberations take place between the interface and the end wall. The same terminology is used for the heavy/light interfaces [Fig. 2(b)], for which all reverberating waves are compression waves.

Experimental wave diagrams obtained from high-speed motion picture and pressure transducer records are compared with those calculated using the simple one-dimensional gas dynamics theory; good agreement is obtained, which is used to confirm the purity of the test gas and to ensure that no leakage was present at the interface before a run.

\section{BOUNDARY LAYER PHENOMENA}

In all experimental studies of the Richtmyer-Meshkov instability, a viscous boundary layer develops in the fluid behind the incident shock which deforms the interface near the walls. The interaction of the reverberating waves with this perturbation leads to the formation of various flow features which can both distort the interface in the bulk of the fluid by vorticity-induced strain and enhance the mutual penetration of the two fluids across the interface.

\section{A. Development of a wall boundary layer at the interface}

\section{Observation of a wall boundary layer at the interface}

The impulsive acceleration of the gases behind a shock wave propagating into an initially stationary fluid leads to the formation of a viscous boundary layer in order to satisfy the no-slip boundary condition at the wall. Following the interaction of the incident shock wave with the interface in the Richtmyer-Meshkov instability, this leads to the overall deformation of the interface, schematically shown in Fig. 3. For this case, after the interface has travelled down the tube a distance $L$ from its original position [shown in Fig. 3(a)], the wall boundary layer has grown to a thickness $\epsilon(L)$ at the interface [Fig. 3(b)]. Furthermore, the interface region near the wall comprises a mixture of test gas and air because of diffusion and entrainment promoted by the boundary layer.

The effects of the boundary layer on the interface after interaction with the incident shock wave can be visualized with the schlieren system. For example, Fig. 10(a) shows a continuous air $/ \mathrm{SF}_{6}$ interface at a time $t=3.59 \mathrm{~ms}$ after inter- 


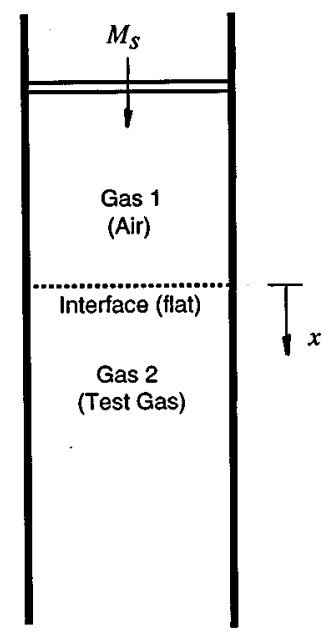

(a)

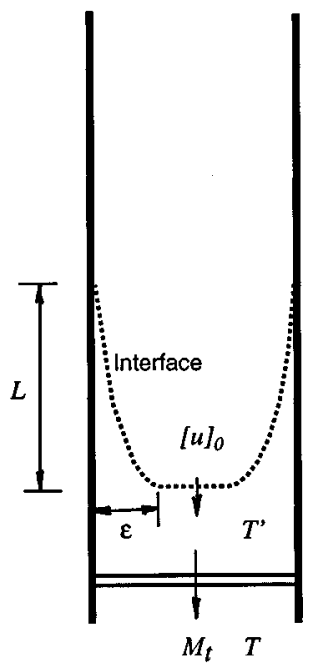

(b)
FIG. 3. Development of boundary layer on side walls following the refraction of the incident shock wave at the interface. (a) Initial configuration. (b) Distorted interface in the presence of a viscous boundary layer, whose thickness is $\epsilon$ at the interface after the latter has travelled a distance $L$ from its initial position.

action with a $M_{s}=1.32$ incident shock; the interface has travelled $38 \mathrm{~cm}$ from its original location. The interface is curved upwards near the wall of the tube under the influence of the boundary layer. The boundary layer thickness can be measured approximately as $\epsilon \approx 4 \mathrm{~mm}$ from the extent of the curved region on the interface; this is only an estimate since, as the tube is square, the boundary layer is tridimensional in the corners of the tube. A summary of measured values for the thickness of the boundary layer in the test gas, when the interface has traveled to the middle of the observing windows $(L=38 \mathrm{~cm})$ following the refraction of the incident shock for a series of long period experiments, is shown in Table I.

\section{Computation of boundary layer thickness at the interface}

The development of a laminar boundary layer behind a shock wave has been treated by many authors (e.g., Mirels ${ }^{8}$ ). In the present study, since the pressure (i.e., Reynolds number) is relatively high and the walls of the shock tube are rough, the boundary layers are likely to be turbulent and the analysis of Mirels ${ }^{9,10}$ is used.

Mirels' analysis assumes that the compressible turbulent boundary layer behind a shock wave obeys a 1/7 power-law velocity profile, and that the gas specific heat ratio $\gamma$ and Prandtl number $P r$ are constant throughout the layer. The latter is a reasonable approximation for the relatively weak shock waves $\left(M_{s}<1.7\right)$ considered in this study. Since the static temperature $T$ is allowed to vary through the boundary layer, the dynamic viscosity $\mu$ of the gas is assumed to follow a $T^{1 / 2}$ temperature dependence, although it is found that the calculated boundary layer thickness is not very sensitive
TABLE I. Comparison of measured and calculated boundary layer thickness at the interface for long period experiments. Also shown is the predicted occurrence of reshock bifurcation in test gas below the interface and in air above the interface; all experimental observations agree with these predictions of bifurcation. The Atwood ratio $A_{0}$ is defined as $A_{0}$ $\equiv\left(\rho_{\text {test }}-\rho_{\text {air }}\right) /\left(\rho_{\text {test }}+\rho_{\text {air }}\right)$, where $\rho_{\text {air }}$ and $\rho_{\text {test }}$ designate the initial density of the air and test gas, respectively.

\begin{tabular}{|c|c|c|c|c|c|c|}
\hline \multirow[b]{2}{*}{ Test gas } & \multirow[b]{2}{*}{$A_{0}$} & \multirow[b]{2}{*}{$M_{s}$} & \multicolumn{2}{|c|}{$\epsilon(\mathrm{mm}) @ L=38 \mathrm{~cm}$} & \multicolumn{2}{|c|}{ Bifurcation of reshock } \\
\hline & & & calculated & measured & in test gas & in air \\
\hline \multirow[t]{4}{*}{$\mathrm{He}$} & -0.76 & 1.32 & 7.8 & 7 & no & yes \\
\hline & & 1.48 & 7.2 & 6 & no & yes \\
\hline & & 1.66 & 6.8 & 6 & no & yes \\
\hline & & $1.66^{\mathrm{a}}$ & 11.3 & 9 & no & yes \\
\hline \multirow[t]{3}{*}{ Air } & 0 & 1.32 & 5.5 & 5 & yes & yes \\
\hline & & 1.48 & 5.0 & 5 & yes & yes \\
\hline & & 1.66 & 4.8 & 5 & yes & yes \\
\hline \multirow[t]{4}{*}{$\mathrm{R}-22$} & 0.50 & 1.12 & 5.0 & 5 & no & no \\
\hline & & 1.32 & 4.2 & 5 & yes & no \\
\hline & & 1.48 & 3.8 & 3 & yes & no \\
\hline & & 1.66 & 3.5 & 3 & yes & no \\
\hline \multirow{4}{*}{$\mathrm{SF}_{6}$} & 0.67 & 1.12 & 4.8 & 4 & no & no \\
\hline & & 1.32 & 3.9 & 4 & yes & no \\
\hline & & 1.48 & 3.5 & 4 & yes & no \\
\hline & & $1.66^{\mathrm{a}}$ & 5.3 & 4 & yes & no \\
\hline
\end{tabular}

${ }^{\mathrm{a}} L=71 \mathrm{~cm}$.

to the prescribed viscosity power-law temperature dependence for the shock strengths under consideration here.

Of primary interest is the determination of the boundary layer thickness $\epsilon$ in the test gas at the interface, after the latter has traveled a distance $L$ following the arrival of the incident shock (Fig. 3). The expression for $\epsilon$ in the test gas behind the transmitted shock is given by:

$$
\begin{aligned}
\frac{\epsilon}{L}= & 0.05745[0.125+S(V-1)]^{-4 / 5} \\
& \times\left[\frac{T^{\prime}}{T}\right]^{4 / 5}\left[\frac{T_{m}}{T}\right]^{-1 / 2}\left[\frac{[u]_{0} L}{\nu}\right]^{-1 / 5},
\end{aligned}
$$

where

$$
V=\left[1-\frac{2}{\gamma+1}\left(\frac{M_{t}^{2}-1}{M_{t}^{2}}\right)\right]^{-1 / 2}
$$

is the so-called velocity parameter, and the temperature ratios of interest are obtained from

$$
\begin{aligned}
& \frac{T_{m}}{T}=0.5\left(1+\frac{T^{\prime}}{T}\right)+0.22\left(\frac{T^{\prime}}{T}\right)\left(\frac{T_{r}}{T}-1\right) \\
& \frac{T_{r}}{T}=1+\frac{\operatorname{Pr}^{1 / 3}(V-1)^{2}}{\frac{\gamma+1}{\gamma-1} V-1},
\end{aligned}
$$

and $T$ and $T^{\prime}$ are the static temperatures in the bulk of the test gas ahead and behind the transmitted shock, respectively, $T_{m}$ is the mean temperature in the boundary layer and $T_{r}$ is the recovery temperature. The interface velocity imparted by the refraction of the incident shock wave is $[u]_{0}$, and $\nu, \gamma$, and $P r$ are, respectively, the kinematic viscosity, the specific heat ratio, and the Prandtl number in the test gas 
TABLE II. Turbulent boundary layer parameter $S$ in the evaluation of the boundary layer thickness in the test gas at the interface [cf. Eq. (1)].

\begin{tabular}{ccccccc}
\hline \hline Gas & Air & $\mathrm{CO}_{2}$ & $\mathrm{R}-22$ & $\mathrm{Xe}$ & $\mathrm{SF}_{6}$ & $\mathrm{He}$ \\
\hline$S$ & 0.039 & 0.036 & 0.033 & 0.045 & 0.031 & 0.045 \\
\hline \hline
\end{tabular}

ahead of the transmitted shock. The parameter $S$ and the numerical constant 0.125 in Eq. (1) are obtained from a straight line fit to a numerical integration; the values of the parameter $S=S(\gamma, P r)$ are listed in Table II for the different gases under consideration in the present study. The constant 0.05745 is derived from an empirical relation for turbulent boundary layers. ${ }^{9,10}$ This analysis ignores the interaction of waves initially reflected from the interface with the boundary layer above the interface and also assumes that the boundary layer comprises only test gas.

Table I compares the calculated and observed values for the thickness of the boundary layer in the test gas when the interface has traveled to the middle of the observing windows $(L=38 \mathrm{~cm})$ following the refraction of the incident shock for a series of long period experiments; there is good agreement between the calculated and observed thickness of the boundary layer at the interface. Since the square test section has a side of $114 \mathrm{~mm}$, the boundary layer at the interface occupies between 5\% and 15\% of the width of the test section for the present experiments which are performed at atmospheric initial pressure.

\section{B. Interaction of reshocks with the boundary layer}

The propagation of the reshocks into a flow field distorted by the development of a wall boundary layer leads to a variety of flow phenomena dominated by shock refraction and vorticity generation mechanisms. These flow features are different in the regions below, above, and just at the interface.

\section{Reshock propagation below the interface}

The propagation in the test gas of the first reshock before it reaches the interface is characterized by the fact that, below the interface, the boundary layer fluid has the same composition as that outside the boundary layer, i.e., they both contain pure test gas. Under certain conditions, the reshock can be observed to bifurcate as a result of its interaction with the boundary layer, and for this case a "bubble" of fluid can accumulate at the base of the reshock. For example, Fig. 4 shows the bifurcation of the first reshock in air, which was the test gas for this particular experiment. The bifurcation bubble is the dark region at the base of the shock, and it can be seen that it has about the same thickness as that of the boundary layer $(\epsilon \approx 5 \mathrm{~mm})$.

The bifurcation of the reshock in the test gas below the interface can be explained in terms of the so-called Mark bubble. Specifically, Mark ${ }^{11}$ has studied the shock-boundary layer interaction for a shock wave reflecting from the end wall of a shock tube [Fig. 5(a)]. Figure 5(b) shows a schematic for the interaction; the analysis is performed more easily in shock-fixed coordinates [Fig. 5(c)].

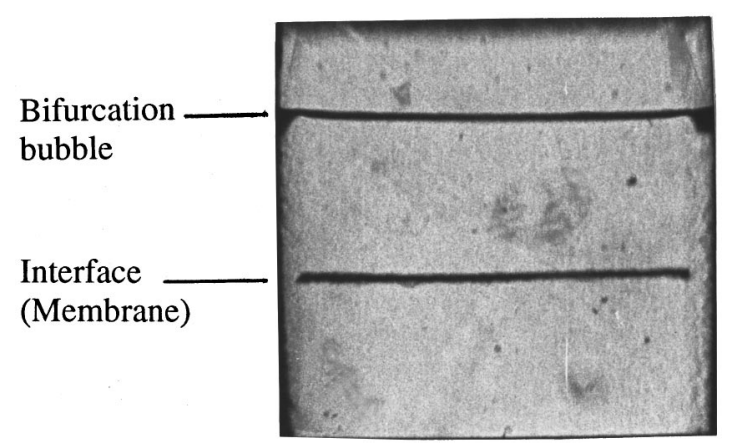

FIG. 4. Interaction of a nitrocellulose membrane (air/air interface) with a $M_{s}=1.32$ shock wave, long time configuration, $t=2.56 \mathrm{~ms}$. Schlieren photograph from high-speed motion picture shows extent of boundary layer below, above and at the interface and also shock wave/boundary layer interaction (Mark bubble) of first reshock.

From the velocities obtained in shock-fixed coordinates, Mach numbers characterizing the outer flow $\left(M_{\text {out }}\right)$ and the boundary layer flow closest to the wall $\left(M_{b l}\right)$ can be defined; in particular, if the boundary layer flow is supersonic (i.e., if $\left.M_{b l}>1\right)$ a shock forms in the boundary layer. Mark proposed that when the stagnation pressure in the boundary layer fluid $p_{T_{b l}}$ exceeds the static pressure behind the reflected shock $p^{\prime \prime}$ then the boundary layer fluid passes con-
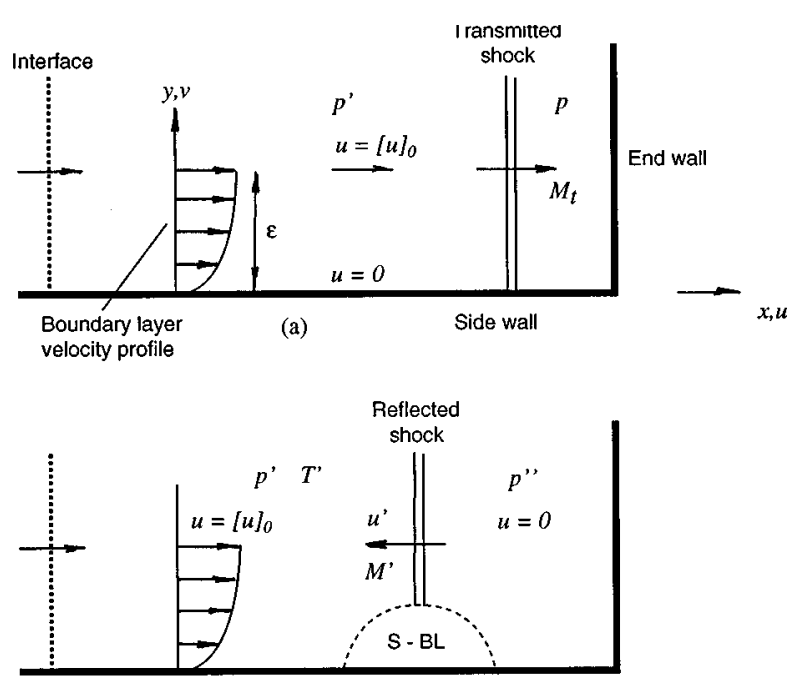

(b)

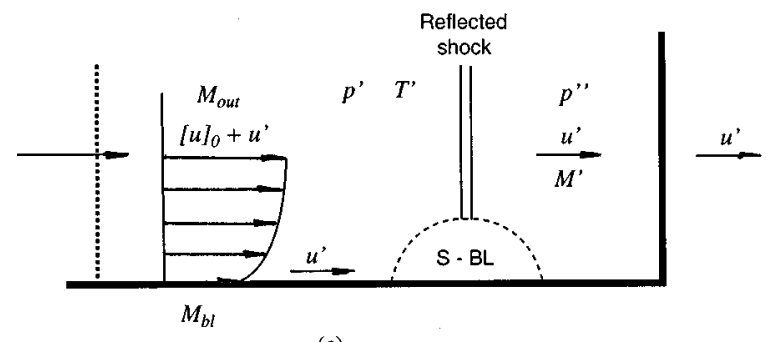

(c)

FIG. 5. Shock wave/boundary layer interaction in test gas: the incident shock wave is travelling from left to right in the reference frame of the laboratory. The region of shock-boundary layer interaction is indicated by "'S-BL.', (a) Shock incident to end wall. (b) Interaction of reflected shock with boundary layer in laboratory reference frame. (c) Same interaction as in (b) but in shock-fixed reference frame. 


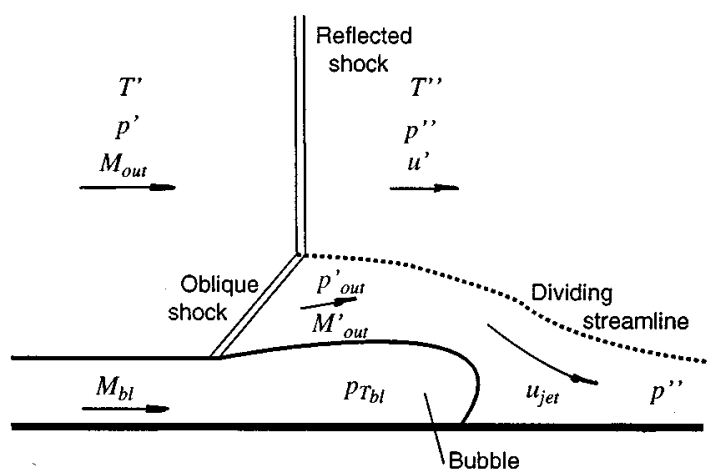

(a)

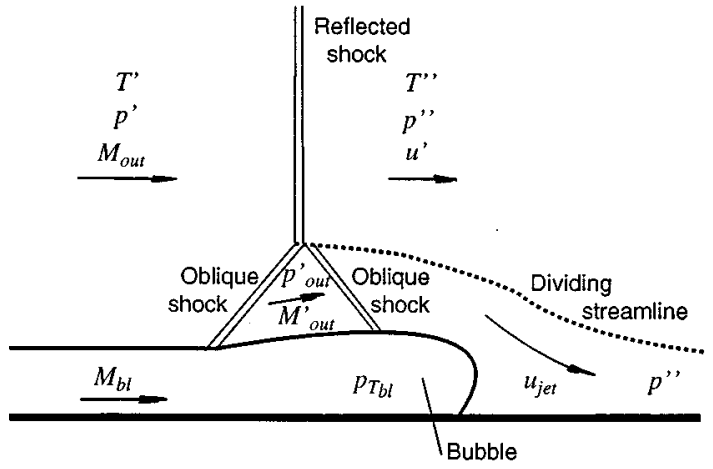

(b)

FIG. 6. Shock system over bifurcation bubble in test gas (Mark bubble) in reference frame stationary with reflected shock. (a) $M_{\text {out }}^{\prime}<1$. (b) $M_{\text {out }}^{\prime}>1$.

tinuously under the foot of the reflected shock and into the region behind it. However, for certain values of the transmitted shock Mach number $M_{t}$, it is possible that $p_{T_{b l}}<p^{\prime \prime}$. For this case, a simple steady through flow of the boundary layer fluid cannot be expected. Rather, Mark proposed that this fluid gathers up as a bubble in a region adjacent to the foot of the shock. Assuming that the ratio of specific heats stays constant throughout the boundary layer. Mark calculated that the bubble will appear if the Mach number of the wave incident onto the end wall is within the interval $1.32<M_{t}$ $<6.45$ for diatomic gases $(\gamma=7 / 5)$ and within $1.57<M_{t}$ $<2.80$ for monatomic gases $(\gamma=5 / 3)$. A more refined analysis ${ }^{12}$ takes into account the possibility for the stagnation pressure minimum to occur within the boundary layer away from the wall, in which case the predicted bifurcation range for monoatomic gases is reduced. This explains the general conception that shock bifurcation can be avoided by using monoatomic gases. Also, since the value of $\gamma$ in diatomic gases can decrease as a result of the temperature rise across the shock, the interval of bifurcation is effectively increased for these gases. Polyatomic gases, with their low specific heat ratios, have an even larger interval of bifurcation.

In addition, the disturbance produced by the Mark bubble causes at least one oblique shock to form at the base of the reflected wave (Fig. 6). The turning angle of the first oblique wave can be calculated by assuming that the static pressure $p_{\text {out }}^{\prime}$ behind it is equal to the stagnation pressure in the bubble $p_{T_{b b}}$. If the flow behind this wave is subsonic (i.e., $M_{\text {out }}^{\prime}<1$ ), the necessary increase in pressure after the oblique shock is accomplished by a streamtube area change [Fig. 6(a)]. If $M_{\text {out }}^{\prime}>1$, another oblique shock forms to bring the static pressure up to $p^{\prime \prime}$, the pressure behind the reflected wave [Fig. 6(b)]. Because the flow of the jet over the bubble is processed by one or two oblique shocks as compared to a single normal shock for the flow in the bulk of the fluid, the velocity of the jet $u_{\text {jet }}$ is therefore larger than that of the main flow behind the reflected shock $u^{\prime}$, with a dividing streamline (i.e., a shear layer) separating the two streams.

Table I lists the predicted occurrence of bifurcation of the reshock in the test gas to form a Mark bubble for a series of experiments with $\mathrm{He}, \mathrm{R}-22, \mathrm{SF}_{6}$, and air as test gases. All of these predictions are in agreement with the experimental results.

\section{Reshock propagation above the interface}

The propagation of the reshock above the interface is complicated by the fact that the fluid within the boundary layer does not have the same composition as that outside of it. For the present experiments, the fluid outside the boundary layer above the interface is air and that inside the boundary layer is presumably a mixture of both air and test gas, as discussed before. This difference in composition leads to a mismatch in speed of sounds which can then promote shock bifurcation.

As an example, Fig. 7 shows the propagation of the reshock across an air/He interface for a long period experiment. The result of the interaction of the reshock with the boundary layer at the interface leads to the formation of a complex bifurcated shock system which is trailed by a large bubble. Specifically, since the boundary layer fluid has a higher speed of sound (i.e., it contains helium) than that of the air outside the boundary layer, a precursor wave forms ahead of the transmitted shock.

These flow features can be explained in terms of the so-called Hess ${ }^{13}$ bubble. The criteria for the appearance of a bifurcated wave and associated bubble are based on the same pressure-matching arguments as for the Mark bubble, although in this case the first oblique shock over the bubble can be viewed as a precursor wave. In addition, Hess proposed that the jet of fluid over the bifurcation bubble can penetrate into the other fluid across the interface but also possibly back under the boundary layer fluid (Fig. 8). This mechanism has in fact been used to explain the contamination of hot test fluid by cold driver gas in reflected shock tunnels. ${ }^{14,15}$

Figure 9 shows a construction, performed using this approach, for the bifurcation of the first reshock as it crosses an air/He interface ( $M_{s}=1.66$, long period experiment). The actual shape of the bubble cannot be determined exactly with the simple arguments presented above; full numerical simulations ${ }^{16}$ have been used to solve this type of problem in more detail. It is seen that there is good agreement between 

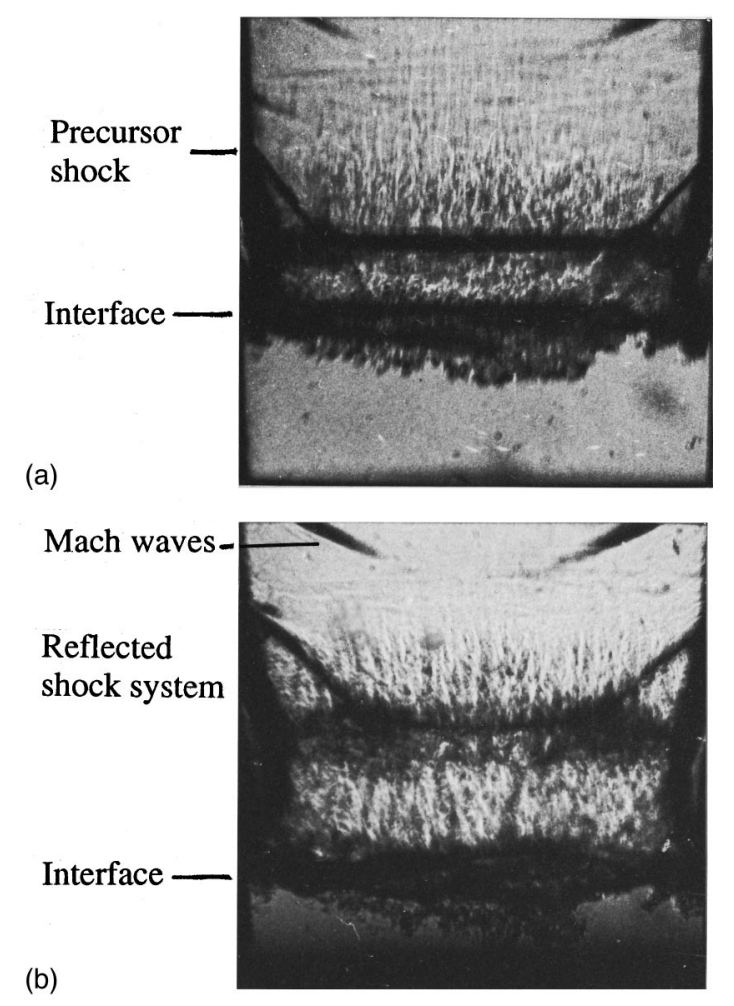

FIG. 7. Richtmyer-Meshkov instability of a plane continuous interface. Air/He $M_{s}=1.66$. Long period experiment. Schlieren photographs from high-speed motion picture. (a) $t=0.86$, (b) $0.91 \mathrm{~ms}$.

the angles obtained from the theoretical construction of Fig. 9 and the experimental results of Fig. 7(b) for the wave pattern at the base of the shock.

For all the cases where bifurcation took place in air above the interface, the calculated velocity of the jet $u_{\text {jet }}$ over the bubble was not much faster than that of the flow behind the reshock $u^{\prime}$, so that contamination of the interface was neither predicted, nor observed. Table I lists the predicted occurrence of formation of a Hess bubble in air after the first reshock crosses the interface from below and these results are also in agreement with the experimental observations.

As seen from Table I, bifurcation of the reshock in air above the interface is not predicted for experiments with the

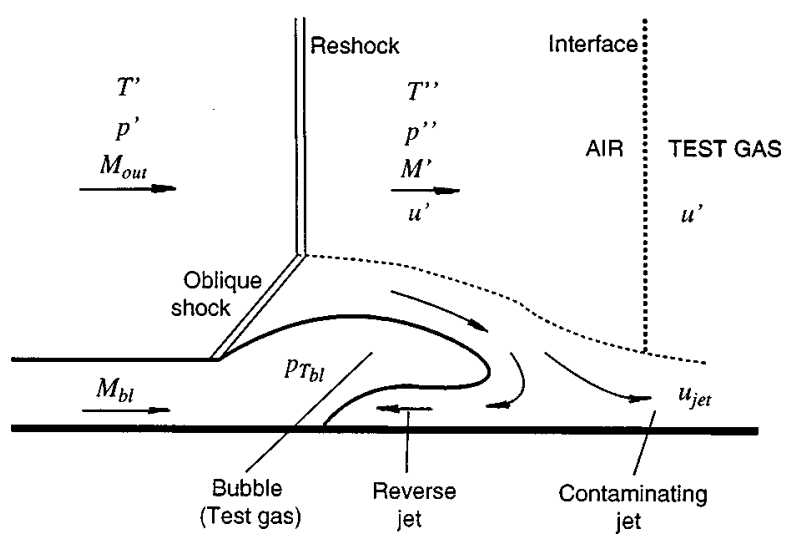

FIG. 8. Formation of contaminating and reverse jets over bifurcation bubble in air after reshock crosses interface (Hess bubble).

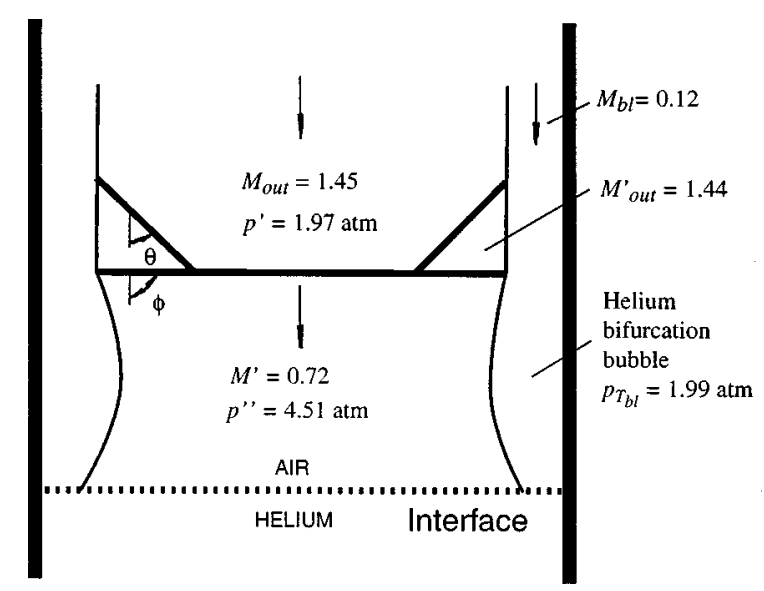

FIG. 9. Theoretical construction for bifurcation wave pattern above interface (in shock-fixed reference frame). Air/He interface, $M_{s}=1.66: \theta=44^{\circ}$, $\phi=87^{\circ}$.

light/heavy interfaces. Thus the Mark-Hess bubble jet phenomenon can neither be used to explain the formation of wall structures at the interface for the Richtmyer-Meshkov instability of light/heavy interfaces nor can it explain the observed contamination of test gas by air for those experiments.

\section{Reshock interaction with the interface}

In addition to the previous two wall phenomena resulting from the interaction of the reshock with the boundary layer below and above the interface, the latter itself can become grossly distorted as a result of its interaction with the reshock. For example, Figs. 10 and 11 show the late time development of light/heavy continuous interfaces for long and short period experiments, respectively. The dominating features of the flow for these cases are large wall vortices that lead to the overall deformation of the interface.

The occurrence of these wall vortices can be explained in terms of baroclinic vorticity generation resulting from the interaction of reshocks with the interface distorted by the wall boundary layers. This can be seen from the schematics of Fig. 12. In Fig. 12(a), a light/heavy interface is shown just before interaction with the first reshock; the interface is distorted at the wall, under the influence of the boundary layer, as it convects down the tube. Because of this interface deformation, the density gradient across the interface near the wall is misaligned with the pressure gradient across the reshock [Fig. 12(a)], and their interaction leads to the deposition of vorticity on the interface in the wall region, as indicated in Fig. 12(b). The subsequent overall deformation of the interface under this vorticity field leads to the roll-up of vortical features on the side walls, as sketched in Fig. 12(c) and observed in Figs. 10(b), 10(c), and 11. Since the tube is actually square, these vortices would develop on all four sides of the test section, with 3D features evolving near the corners; however the arguments presented here would still remain essentially valid.

For the case of heavy/light interfaces, the interface distortion caused by the boundary layer would be similar [Fig. $12(\mathrm{a})]$, but the direction of the density gradient across the 

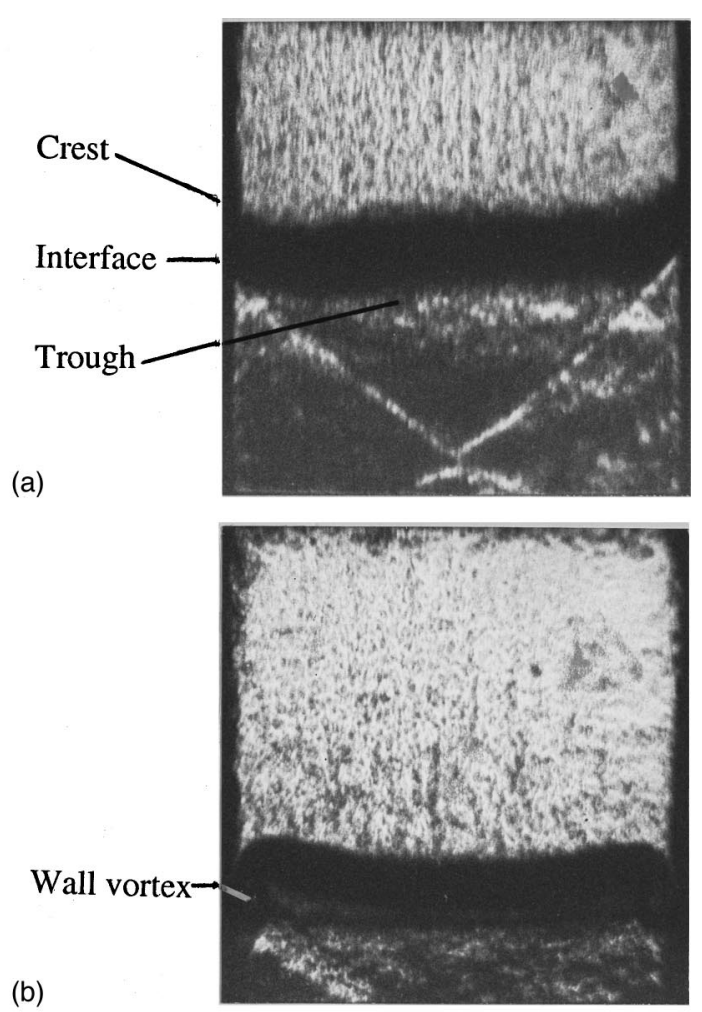

(b)

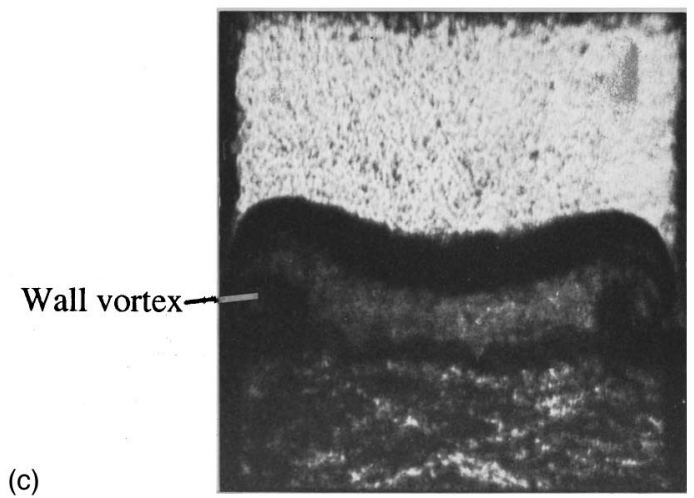

FIG. 10. Richtmyer-Meshkov instability of a plane continuous interface. Air/ $\mathrm{SF}_{6} M_{s}=1.32$. Long period experiment. Schlieren photographs from high-speed motion picture. (a) $t=3.59$, (b) 4.39 , and (c) $5.19 \mathrm{~ms}$. (a)

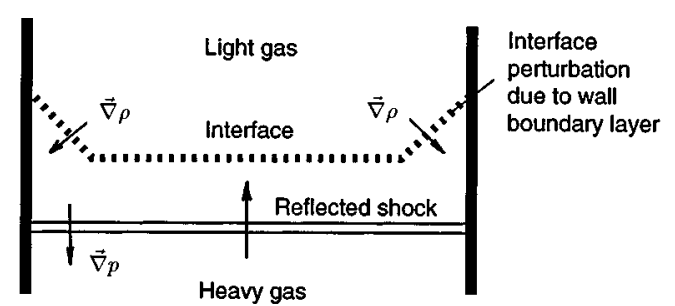

(b)

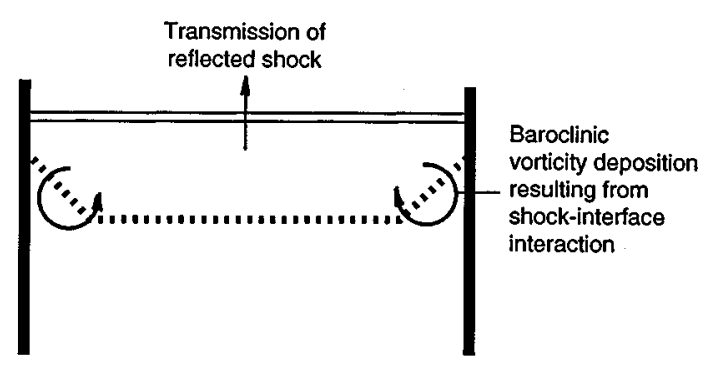

(c)

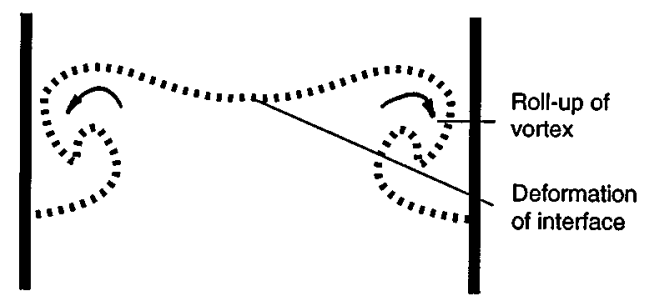

FIG. 12. Mechanism of wall vortex formation on light/heavy interface. (a) Interface perturbation before reshock. (b) Deposition of baroclinic vorticity at interface by reshock. (c) Subsequent roll-up of wall vortex.

interface would be reversed compared to the light/heavy interface. For this case, the interaction of this density gradient with the pressure gradient of the reshock would deposit vorticity in a direction opposite from that shown in Fig. 12(b), and the wall vortex would roll-up in the opposite direction as that shown in Fig. 12(c). However actual results for heavy/ light interfaces (cf. Fig. 7) show that the interface is mostly dominated by a large disturbance resulting from the forma-

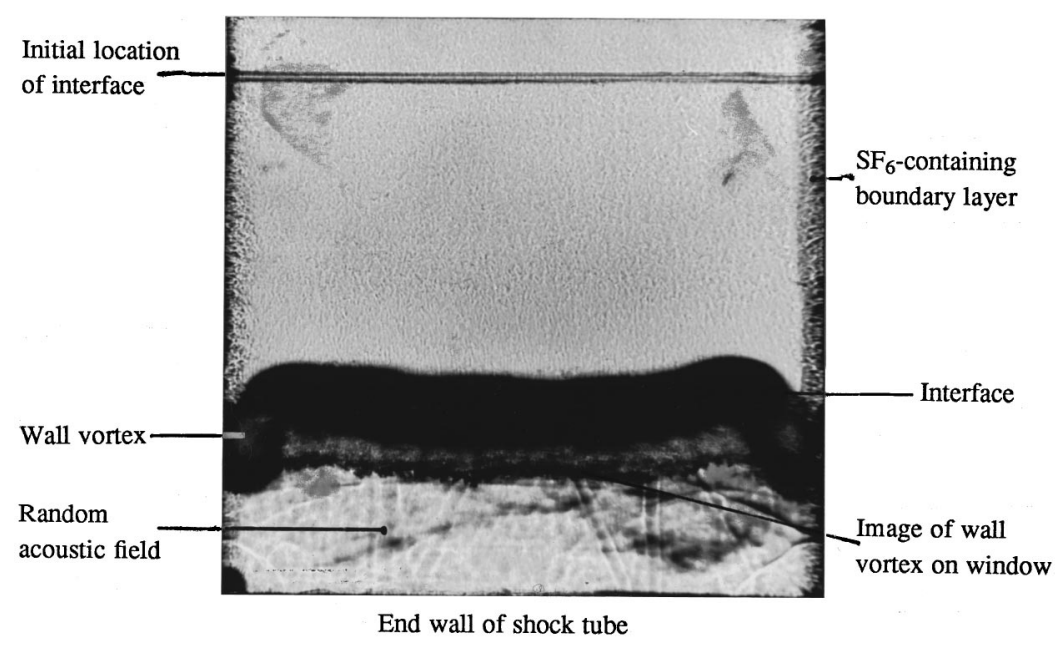

FIG. 11. Richtmyer-Meshkov instability of a plane continuous interface. Air $/ \mathrm{SF}_{6} M_{s}=1.32$, short period experiment. $t=1.91 \mathrm{~ms}$. Spark schlieren photograph. 
(a)

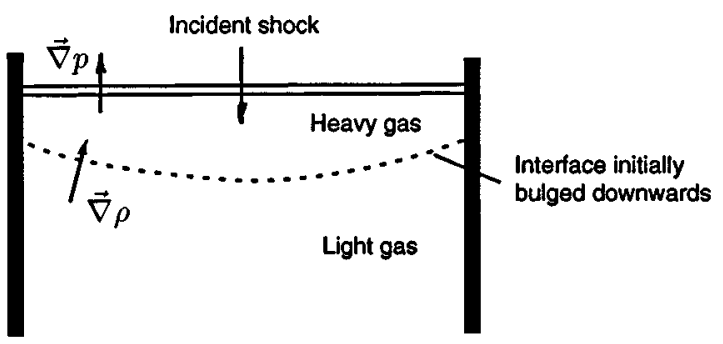

(b)

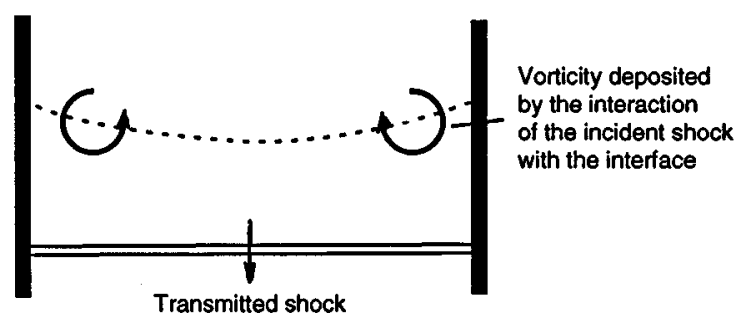

(c)

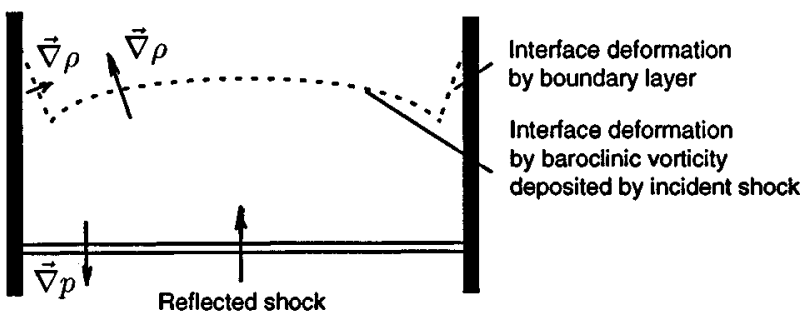

FIG. 13. Mechanism of wall vortex formation on heavy/light interface with an initial downwards bulge. (a) Interface perturbation before incident shock. (b) Deposition of baroclinic vorticity at interface by incident shock. (c) Subsequent deformation of interface after interaction with incident shock. (d) Deposition of baroclinic vorticity at interface by reshock. (e) Subsequent roll-up of wall vortex.

Vorticity deposited by

the interaction of the

reflected shock with the

interface within the boundary layer

Vorticity deposited by

the interaction of the

reflected shock with the interiace

outside the boundary layer

(e)

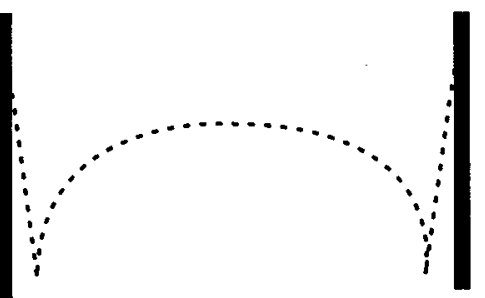

tion the reverse jet under the Hess bubble, since bifurcation takes place above the interface for these cases.

Wall vortices are ultimately the result of the perturbations introduced by the development of a boundary layer at the interface and, for discontinuous interfaces, can be modified by the presence of an initial bulge on the supporting plastic membrane. For example, consider the interaction of the incident shock and subsequent reshocks with a heavy/ light discontinuous interface that is initially bulged downwards (by a small pressure difference across the membrane, for example). Figure 13(a) shows a schematic of the interface just before the interaction with the incident shock. Since the interface is bulged downwards, the initial misalignment of the density gradient across the interface with the pressure gradient across the incident shock leads to vorticity deposition on the interface as shown in Fig. 13(b). Under this vorticity distribution, the interface tends to reverse the phase of the bulge in the upwards direction, while at the same time undergoing deformation on the walls under the influence of the boundary layers [Fig. 13(c)]. When the first reshock interacts with this interface, baroclinic vorticity is deposited as the result of interface deformation both in the bulk of the 

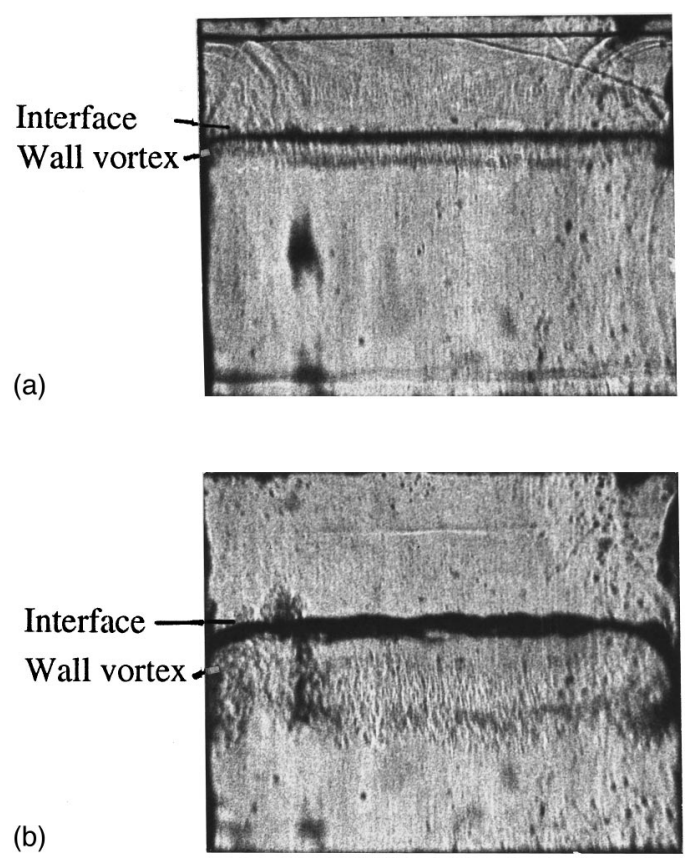

FIG. 14. Richtmyer-Meshkov instability of a plane discontinuous interface. Air/He $M_{s}=1.32$. Short period experiment. Schlieren photographs from high-speed motion picture. (a) $t=0.23$, (b) $0.63 \mathrm{~ms}$.

fluid and within the wall boundary layer, with the former probably dominating the latter since the density gradient across the interface is likely to be larger than within the boundary layer, where mixing between the air and test gas is more important [Fig. 13(d)]. As a result of this deposition of vorticity on the interface, the prereshock interface deformation is then amplified, as shown in Fig. 13(e); it should also be noticed that further interaction of the interface with reverberations from the end wall of the test section just repeat and amplify this process, as all reverberations are shocks for the heavy/light case.

An example of this case is shown in Fig. 14 for a short period experiment with an air/helium interface that was initially slightly bulged downwards. For this case the wall vortex develops as the result of repeated interaction with reshocks reverberating between the interface and the end wall of the tube.

Another case is the light/heavy discontinuous interface that is initially bulged slightly upwards (Fig. 15). For this, the interaction of the incident shock with this interface leads to an increase in the amplitude of the upward bulge. At the same time as the interface propagates down the shock tube and that this bulge grows upwards, the interface is also pulled back on the sides by the action of the wall boundary layer. The resulting perturbation on the interface caused by the combined effect of the bulge and the boundary layer is sketched in Fig. 15(a). Upon the arrival of the reshock, vorticity deposition resulting from interface deformation in the fluid outside the boundary layer dominates, leading to the formation of "reverse" wall vortices [Figs. 15(b) and 15(c)] which roll up in the opposite direction as the vortices on the light/heavy interfaces of Figs. 10 and 11, for which no initial bulge was present. (a)

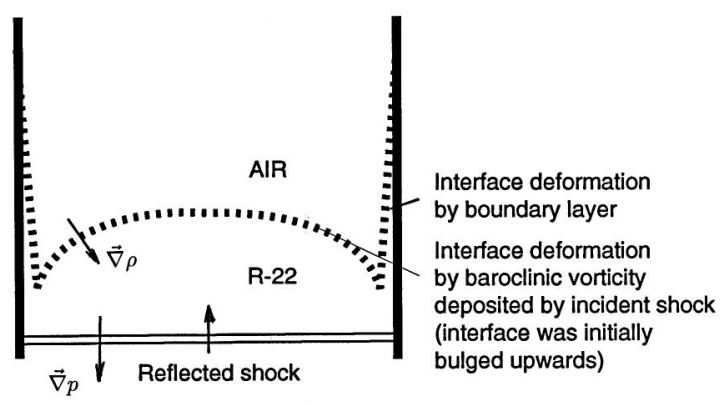

(b)

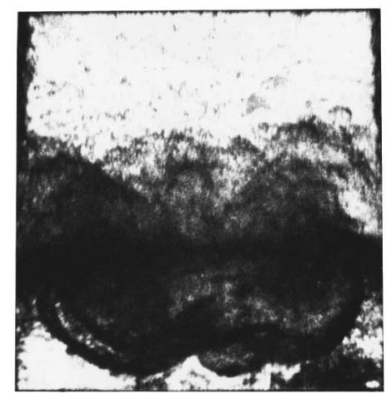

(c)

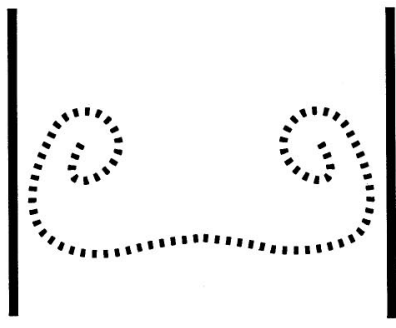

FIG. 15. Reverse wall vortex formation on light/heavy interface with an initial upwards bulge. (a) Deformation of interface after interaction with incident shock. (b) Formation of reverse wall vortex. Air/R-22, $M_{s}=1.32$, long time configuration. Schlieren photograph from high-speed motion picture. (c) Schematic of reverse wall vortex of (b).

\section{WAVE PHENOMENA}

This section discusses issues relevant to the wave motions in the experimental observation and data reduction of the Richtmyer-Meshkov instability. In particular, the influence of the plastic membranes on the initial motion of the interface is examined. Also studied are the two- and threedimensional wave patterns created by the interaction of the planar incident shock wave with a flat interface.

\section{A. Acceleration of the membrane at the discontinuous interface}

The effect of the membrane on the refraction of the incident wave at a discontinuous interface can be estimated by considering the influence of the membrane's inertia. The interaction can be analyzed by modeling the membrane as a rigid piston which does not shatter and whose acoustic impedance is large compared to that of the surrounding gases. It is also assumed that the incident wave does not directly transmit through the membrane but that the refraction of the incident shock at the interface produces a constant acceleration $a=A \Delta p / m$ of the membrane of mass $m$, applied until the interface attains its final velocity $[u]_{0}$. The pressure difference across the membrane is $\Delta p$, and the cross-sectional 
TABLE III. Shock formation distance calculated from Eq. (3) $-M_{s}=1.32$.

\begin{tabular}{ccccccc}
\hline \hline Interface & Air/air & Air/CO & Air/R-22 & Air/Xe & Air/SF & Air/He \\
\hline$d_{\text {shock }}(\mathrm{mm})$ & 0.25 & 0.16 & 0.07 & 0.05 & 0.04 & 2.0 \\
\hline \hline
\end{tabular}

area of the shock tube is $A$. The subsequent motion of the membrane causes the formation of compression waves in the test gas downstream of the interface, and after a certain distance $d_{\text {shock }}$, these waves coalesce into a shock wave. Since the resulting motion of the piston is parabolic in time, a simple expression for the shock formation distance $d_{\text {shock }}$ can thus be obtained as (cf. Thompson ${ }^{17}$ ):

$$
d_{\text {shock }}=\frac{2 m c^{2}}{(\gamma+1) A \Delta p}
$$

where $c$ and $\gamma$ are, respectively, the speed of sound and the specific heat ratio in the unshocked test gas. The pressure difference across the piston $\Delta p$ can be estimated from the pressure behind the reflection of the incident shock from a rigid end wall, since the acoustic impedance of the membrane is assumed to be very large. Table III lists the shock formation distance for six interfaces, calculated from Eq. (3) for an incident shock Mach number $M_{s}=1.32$ at atmospheric initial conditions. The shock formation distance was also computed using a more physically accurate model which took into account the rarefaction waves behind and the compression waves ahead of the membrane as it starts to move into the test gas, but, because of the small inertia of the membrane, the results are almost identical to those obtained with the simplified model of Eq. (3).

It can be seen from those results that, for a given incident shock, the shock formation length increases as the speed of sound of the test gas is increased, i.e., as the molecular weight is decreased. It is then expected that the $1 \mathrm{D}$ gas dynamics theory is valid when the waves emerging from the interface are at least a distance $d_{\text {shock }}$ away from the interface. Also, since $\Delta p \sim p M_{s}^{2}$, where $p$ is the initial pressure in the test section, the shock formation length decreases as the strength of the incident wave is increased. On the other hand, reducing the initial pressure causes $d_{\text {shock }}$ to increase. Therefore, the use of a light test gas at low initial pressure with weak shocks can lead to an unacceptably long shock formation length. For example, for an air/He interface with $M_{s}$ $=1.32$ at $p=0.01 \mathrm{~atm}$, we calculate $d_{\text {shock }}=200 \mathrm{~mm}$, and with $M_{s}=3, d_{\text {shock }}=19 \mathrm{~mm}$, which signifies that the interface acceleration phenomenon is likely to be considerably affected by the presence of the membrane at those conditions. ${ }^{18}$

\section{B. Two- and three-dimensional wave patterns}

The propagation of the interface and waves in the shock tube and test section is not necessarily one-dimensional owing to the presence of boundary layers, possible nonuniformities on the side walls of the tube and small disturbances in the test section.

The refraction of the incident shock at the interface illustrates some of these influences. Figure 16 shows spark
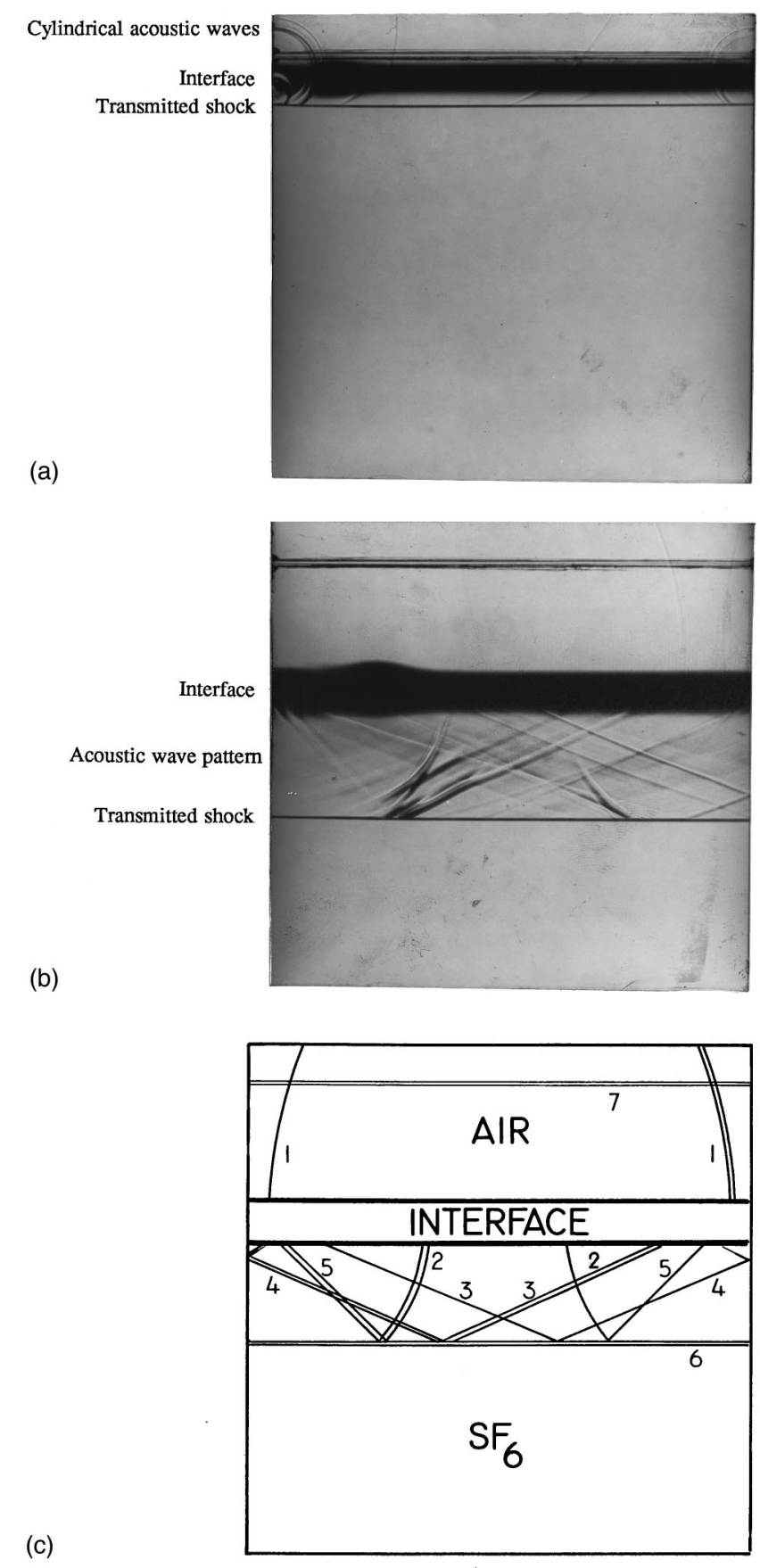

FIG. 16. Wave pattern resulting from the refraction of a $M_{s}=1.32$ shock wave with a air $/ \mathrm{SF}_{6}$ continuous interface. (a) $t=0.04 \mathrm{~ms}$. Spark schlieren photograph. (b) $t=0.27 \mathrm{~ms}$. Spark schlieren photograph. (c) Theoretical wave front construction: 1-Cylindrical wave fronts in air. 2-Cylindrical wave fronts in $\mathrm{SF}_{6}$. 3-Precursor wave fronts in $\mathrm{SF}_{6}$. 4-Reflection of precursor off transmitted shock. 5-Reflection of cylindrical wave fronts off transmitted shock. 6-Transmitted shock. 7-Grease marks left on window by sliding plate.

schlieren photographs of the air/ $\mathrm{SF}_{6}$ interface, initially located in the field of view of the windows. The interface is accelerated by a $M_{s}=1.32$ shock wave just after retraction of the sliding plate, as evidenced by the presence on the left part of the interface of the accumulation of fluid pumped by the plate [Fig. 16(a)]. The photograph shows the interface just after the arrival of the incident shock. Because of the 


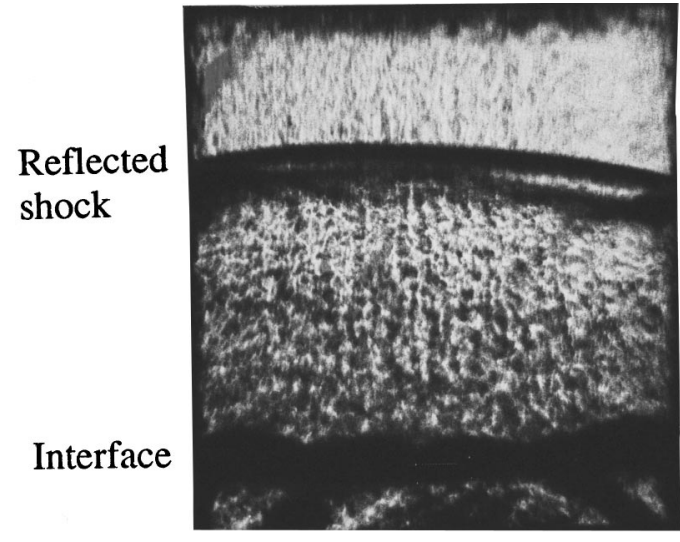

FIG. 17. Richtmyer-Meshkov instability of a plane continuous interface. Air $/ \mathrm{SF}_{6} \quad M_{s}=1.32$. Long period experiment, $t=4.30 \mathrm{~ms}$. Schlieren photograph from high-speed motion picture.

presence of small disturbances on the side walls of the tube, such as joints for the plate guide plug and grease left from the edges of the sliding plate, the reflected and transmitted shocks emerging from the interface are accompanied by cylindrical acoustic waves that originate from the wall at the interface. Another wave front also appears on the left side because of the perturbation introduced by the slot into which the plate is retracted. Since the speed of sound is higher in air than in $\mathrm{SF}_{6}$, the wave fronts propagate faster above the interface. This leads to the generation of precursor waves below the interface [Fig. 16(b)]. Furthermore, the cylindrical wave in $\mathrm{SF}_{6}$ propagates faster downstream than the transmitted shock, and the two interact leading to another reflection. Since the angle between the cylindrical wave front and the shock remains constant in time as they propagate downstream, the weak reflected wave is straight and remains at a constant angle. In this case the parameters are such that this reflection comes back to the interface where it meets the wall. Figure 16(c) is a construction for the shocks, the acoustic waves, and their reflections, with the velocities calculated with the simple 1D gas dynamics theory, for the interface of Fig. 16(b) at the same time interval after the interaction with the incident shock. As can be seen, there is good agreement between the experimental and calculated results.

The result of the interaction of the reshock with the boundary layer above the interface is another example of the two dimensionality of the wave pattern even if shock bifurcation is absent. This is because the boundary layer above the interface is composed mainly of test gas and that its local speed of sound differs from that of the air in the bulk of the fluid. After the reshock crosses the interface, the wave transmitted into air has a different velocity than that propagating into the boundary layer. For the light/heavy interface the transmitted shock is bowed upwards, as seen in Fig. 17, because the speed of sound in the boundary layer is lower. For the case of a heavy/light interface, the transmitted shock is preceded by a precursor shock, as seen in Fig. 7.

Also, when the interface velocity is supersonic in the reference frame of the shock tube with respect to one of the gases adjacent to the interface, small disturbances on the walls of the test section cause the appearance of Mach waves, as seen in Fig. 7(b). For this example, the Mach number of the air flow above the interface is $M=1.10$ in the reference frame of the test section, corresponding to a Mach angle of $65^{\circ}$, which agrees with the observed value.

Finally, when the transmitted shock reflects from the end of the shock tube, cylindrical acoustic waves are also generated from the corners at the end of the shock tube because of the disturbance caused by the displacement thickness of the boundary layer.

For short period as well as long period experiments, the reverberation between the end wall and the interface of the trapped waves and their subsequent interaction produces three-dimensional wave patterns. The end result of this is the randomization of the wave field below the interface, as shown in Figs. 10(c) and 11. The interaction of these waves with the interface is the suggested mechanism for introducing perturbations on the smooth continuous interfaces, the growth of which has been the subject of previous investigation. ${ }^{3,4,19}$

\section{DISCUSSION}

\section{A. Effect of reduced initial pressure}

Equation (1) shows that the turbulent boundary layer thickness in the test gas at the interface varies as $\epsilon_{\text {turb }}$ $\sim[u]_{0}^{-1 / 5} L^{4 / 5} / \nu^{-1 / 5}$, but since $\nu=\mu / \rho$ and $\rho \sim p / T$, it follows that, for a given initial temperature $T, \epsilon_{\text {turb }} \sim p^{-1 / 5}$; for a laminar boundary layer, which would be present for the case of smooth walls and/or low initial test pressure, this power law dependence would become $\epsilon_{\mathrm{lam}} \sim p^{-1 / 2}$. These relationships show that reducing the initial test pressure significantly increases the boundary layer thickness at the interface. In particular, all other parameters remaining constant, performing the experiments at initial pressures of $p=0.1,0.01$, and $0.001 \mathrm{~atm}$, for example, would increase the turbulent boundary layer thickness at the interface by factors 1.6, 2.5, and 4.0, respectively, with respect to experiments at atmospheric conditions; for a laminar boundary layer at these pressures, this thickness would be increased by factors of 3.2, 10, and 31 , respectively, over experiments performed at $1 \mathrm{~atm}$. For the present experimental facility, this would mean that reducing the initial pressure in the test section, for example to increase the incident shock Mach number, could produce boundary layers at the interface which could occupy up to $50 \%$ of the width of the test section; this would certainly have a severe influence on the development of the instability at the interface. In particular, this argument explains the highly distorted shape of interfaces observed by Houas et al. ${ }^{18}$ in their experimental study of the RichtmyerMeshkov instability performed at low pressure $(0.015 \mathrm{~atm})$ with a narrow test section $(8.5 \mathrm{~cm}$ square section).

Large boundary layers not only influence the natural development of the instability at the interface following the refraction of the incident shock, but greatly modify the extent of the shock-boundary layer region after the first and subsequent reshocks. In particular, it is generally agreed that the width of the Mark or Hess bubbles resulting from shock bifurcation scales directly with the thickness of the boundary layer at the interface (e.g., Fig. 4), and the present results 
also show that the width of the wall vortex also seems to scale directly with boundary layer thickness. For example, for shocks of modest strength $\left(M_{s} \approx 1.5\right)$ and interfaces between gases initially at atmospheric pressure with density ratios of order $2-5$, the wall vortex on each side wall was seen to extend up to about $2 \mathrm{~cm}$ into the bulk of the fluid, occupying about $35 \%$ of the width of the test section and $60 \%$ of its cross-sectional area. These simple pressure scaling arguments point out that, for experiments that reduce the initial pressure down to only $0.1 \mathrm{~atm}$, the wall vortex could be 1.6 times larger, if the boundary layer were turbulent, and the wall vortex could therefore occupy up to $60 \%$ of the width and $80 \%$ of the area of the test section. This effect would be even more catastrophic in the presence of a laminar boundary layer.

Also, as seen from Eq. (3), the influence of the membranes used to form initially discontinuous interfaces is strongly dependent on initial test pressure. In particular, the so-called shock formation distance scales as $d_{\text {shock }} \sim p^{-1}$. As shown before, the shock formation distances for the present experiments performed at $1 \mathrm{~atm}$ are estimated to be of the order of $1 \mathrm{~mm}$; decreasing the initial pressure to 0.1 or 0.01 atm would therefore increase these distances to 1 or $10 \mathrm{~cm}$, respectively. When the magnitude of $d_{\text {shock }}$ approaches the other relevant length scales in the problem, such as test section diameter, interface amplitude, or wavelength, for example, the shock refraction phenomena at the interface is likely to be greatly influenced by the presence of the membrane.

The effects of initial pressure on shock wave-boundary layer phenomena at the interface and on the influence of membranes greatly underscore the need to perform experiments at high initial test pressures with large test sections. These conditions sometimes have to be compromised, however, because of the high cost associated with large facilities, the experimental difficulties inherent with the use of large membranes, which require a supporting wire mesh that introduces further perturbations, ${ }^{20}$ and the need for reduced pressures to generate high incident shock Mach numbers.

\section{B. Interpretation of flow visualization results}

The present results also emphasize the importance of using flow visualization methods that can distinguish between wall boundary layer effects and the interface in the bulk fluid. Any uncertainty about the real extent of the turbulent mixing zone (TMZ) evolving at the interface will be cleared only if at least one side wall is imaged along with the phenomena of interest in the bulk of the fluid. Failure to distinguish between TMZ and wall effects always lead to overestimated growth rates. ${ }^{3,4}$ In particular, the streak schlieren method does not seem to be appropriate for TMZ growth studies since it fails to image either the TMZ or the side walls. Qualitative imaging methods such as shadowgraph or schlieren photography are adequate for the task, but are handicapped by the somewhat interpretative judgement that has to be applied in the data reduction.

Quantitative imaging methods seem naturally better suited to accomplish this task. However, when using a tech- nique that relies on an integration across the depth of the test section, such as interferometry or x-ray densitometry, ${ }^{7,21}$ for example, some care has to be exercised in the data reduction to correct for the wall effects.

Figure 18(a) shows the optical density of a radiograph taken at $t=5.82 \mathrm{~ms}$ after the interaction of a $M_{s}=1.33$ shock wave with an air/xenon interface for a long period experiment. The brightest regions correspond to region of lowest densities, and vice-versa. The roll-up of the wall vortices can clearly be seen and is reminiscent of Fig. 10(c) for a corresponding experiment with an air/SF 6 interface. Figures 18(b), 18(c), and 18(d) show the computed mean interface shape, the density contours and the average density profile for the aforementioned air/xenon interface. As expected from the previous results, the only distortions on the interface are due to the wall vortices, which are also apparent from the density contours [Fig. 18(c)]. Because of Lambert's law, the observed optical density field is the result of an integration of the local density across the depth of the test section between the flow visualization windows. By proper calibration, the average density field across the depth of the test section can thus be obtained from the optical density field. Because wall vortices develop on all four walls of the test section and contain a mixture of air (which has negligible x-ray absorption) and xenon, even the density profiles obtained in the middle of the test section are modified by the wall vortices on the windows, as seen from Fig. 18(d). The results can be partially corrected for the undesirable contribution of the wall vortices on the windows by modifying the observed optical density near the side walls such that the optical density at a given axial location averaged over the width of the test section is the same as that averaged in the bulk of the fluid. $^{7,21}$ Using the same approach, a similar correction method could be applied for extracting density information from interferometer images.

Because of these limitations, it appears that planar (i.e., sheet forming), flow visualization methods offer the best prospects for eliminating the qualitative and/or quantitative ambiguities associated with the aforementioned methods. For example, planar laser-induced fluorescence (PLIF) was used by Jacobs ${ }^{22}$ to study the Richtmyer-Meshkov instability in a cylindrical geometry. The experimental difficulty there was the dissimilar properties of the test gas and the fluorescent tracer gas; future experiments could use PLIF without resorting to tracers altogether. Another promising avenue is the use of Rayleigh scattering. ${ }^{23}$

\section{Interpretation of measured growth rates}

For discontinuous interfaces, the effect of the membranes on the measured TMZ growth rates is still to be determined. ${ }^{4,20,24,25}$ Previous long period experiments ${ }^{4}$ with air/air discontinuous interfaces formed by thin $(0.5 \mu \mathrm{m}) \mathrm{ni}-$ trocellulose membranes have shown that, even though these interfaces initially present no density mismatch, some thickening of the membrane region is still observed (cf. Fig. 4). In particular, the reshock growth rates of the interface region for these experiments ranged between 1 and $5 \mathrm{~m} / \mathrm{s}$ and seemed to increase with incident shock Mach numbers. A 


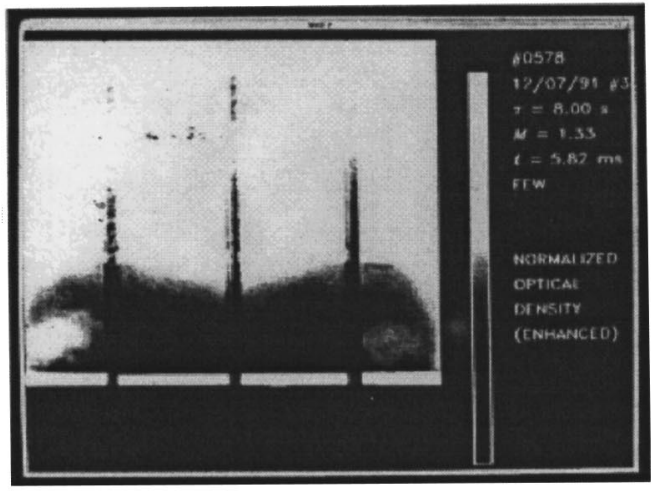

(A)

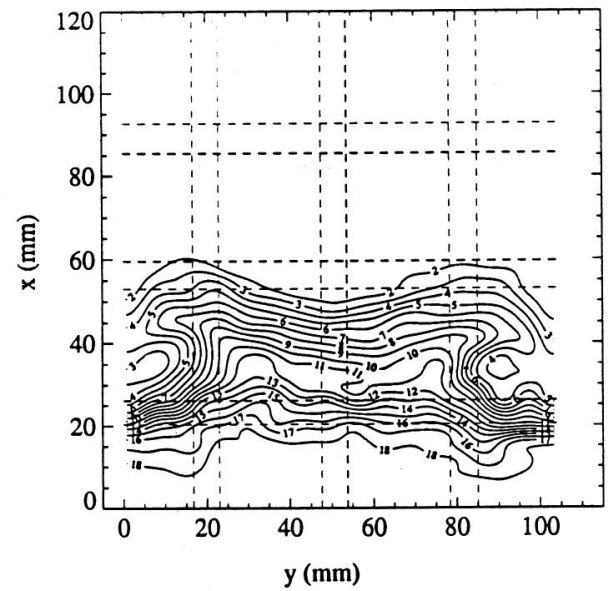

(C)

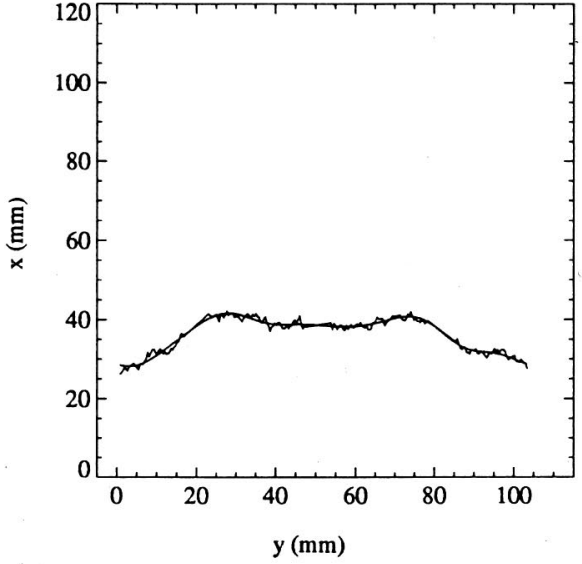

(B)

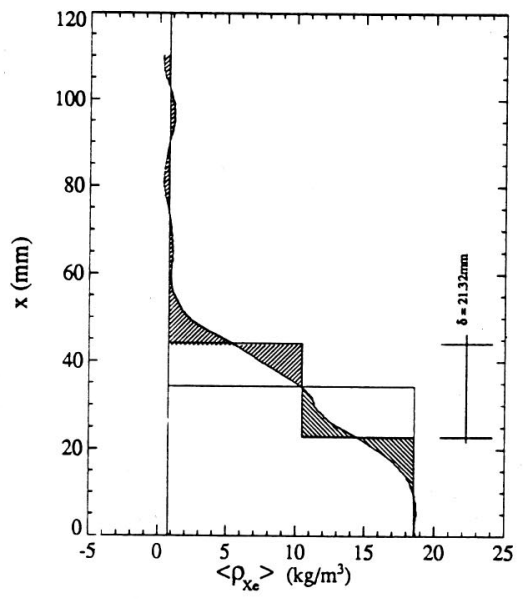

(D)

FIG. 18. Richtmyer-Meshkov instability of a plane continuous interface. Air/xenon, $M_{s}=1.33$. Long period experiment, $t=5.82 \mathrm{~ms}$. (a) Optical density of radiograph. (b) Mean interface shape. (c) Density contours (labeled in $\mathrm{kg} / \mathrm{m}^{3}$ ). (d) Average density profile.

feature of the air/air interfaces is that the membrane did not appear to shatter upon wave refraction at the interface. If this were the case for discontinuous interfaces formed between dissimilar gases, the membranes would have a strong growth-impairing effect; there is no experimental evidence, however, that the membrane does not shatter when the Richtmyer-Meshkov instability is present. Experiments performed at high incident shock Mach numbers would clear this uncertainty as the temperatures generated by the waves could be sufficient to vaporize the plastic membrane. ${ }^{26}$

The development of vortices on the side walls of the test section can seriously modify the thickening of the interfaces caused by the Richtmyer-Meshkov instability in the bulk of the fluid. In particular, the velocity field induced by the wall vortices stretches the interface which causes a reduction in its apparent rate of turbulent thickening. The magnitude of this effect can be estimated by computing the strain field associated with two incompressible line vortices each positioned a distance $D$ from an infinite vertical side wall (Fig. 19). Since the system is periodic in the $y$ direction, ${ }^{27}$ the strain rate $\dot{s}$ produced in the middle of the test section ( $y$ $=W / 2$ ) by these two vortices is given by:

$$
\dot{s}=\frac{2 \pi \Gamma}{W^{2}} \frac{\sinh \left(\frac{2 \pi x}{W}\right) \sin \left(\frac{2 \pi D}{W}\right)}{\left[\cosh \left(\frac{2 \pi x}{W}\right)+\cos \left(\frac{2 \pi d}{W}\right)\right]^{2}},
$$

where $\Gamma$ and $W$ are, respectively, the circulation of each vortex and the width of the test section. The strain rate in the middle of the test section can thus be calculated for any axial location $x$ given the value of $D$ and $\Gamma$.

For example, for a long period experiment performed with a continuous air $/ \mathrm{SF}_{6}$ interface and $M_{s}=1.32$ (Fig. 10), the location of the wall vortices at $t=5.19 \mathrm{~ms}$ is at $D=10$ $\mathrm{mm}$ and the middle of the interface is at $x=-0.20 \mathrm{~mm}$ with respect to the wall vortices. The circulation $\Gamma$ can be estimated by evaluating the tangential velocity $u_{t}$ of the roll-up of the vortex from $\Gamma \approx 2 \pi u_{t} R$, where $R$ is the radius of the vortex. For this particular case, $u_{t} \approx 10 \mathrm{~m} / \mathrm{s}$ and $R \approx 10 \mathrm{~mm}$, 


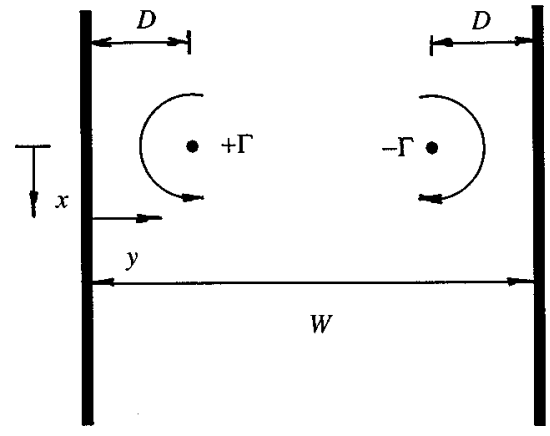

FIG. 19. Schematic of vortex pair configuration used to estimate interface strain induced by wall vortices.

then $\Gamma \approx 0.6 \mathrm{~m}^{2} / \mathrm{s}$, and the strain rate in the middle of the interface is thus found to be $\dot{s} \approx-30 \mathrm{~s}^{-1}$. This is the strain rate induced in the middle of the test section by a pair of opposite wall vortices, but because the test section actually possesses four walls (and thus two wall vortex pairs), the total strain rate $\dot{s}_{\text {tot }}$ in the middle of the test section due to three-dimensional induction is estimated by a simple superposition of the strain rates of both pairs of wall vortices, i.e., $\dot{s}_{\text {tot }} \approx 2 \dot{s}$. For the present example, the total strain rate in the middle of the interface is therefore estimated at about -60 $\mathrm{s}^{-1}$. Since it can be shown for most cases that the value of the strain rate does not vary very much throughout the thickness of the interface, the overall thinning rate of the interface due to wall vortex strain can be estimated from:

$$
\left(\frac{d \delta}{d t}\right)_{\text {vortex }} \approx \delta \dot{s}_{\text {tot }}
$$

where $\delta$ is the observed thickness of the interface. For this example, since $\delta=10 \mathrm{~mm}$, the thinning rate induced by wall vortices is about $0.6 \mathrm{~m} / \mathrm{s}$, which is of the same order of magnitude as the measured overall thickening rate of the TMZ at this interface for this experiment. ${ }^{4}$ Although the actual problem is three-dimensional, this result has the right order of magnitude.

In summary, in addition to grossly distorting the shape of the interface, the wall vortices play a role in the evolution of the thickness of the TMZ at the interface, most importantly when very little growth is observed such as in the smooth continuous interface experiments. For the continuous interface, the thinning rate is estimated at about $50 \%$ of the reshock and late-time growth rates. For discontinuous experiments, where TMZ thickening is more rapid, the magnitude of the wall vortex strain is estimated at no more than $10 \%$ for the experiments performed in the present facility. It is also interesting to note that, when wall vortices are developing below the interface, as it is the case for all the present results, the strain rate at the interface is negative, which reduces the thickening rate of the TMZ; on the other hand, if wall vortices were developing above the interface, as it is the case for reverse wall vortices (Fig. 15), the opposite effect would take place: the growth rate of the TMZ would be increased. Altogether, the influence of wall effects can be reduced by avoiding large boundary layer thicknesses and using wide test sections.

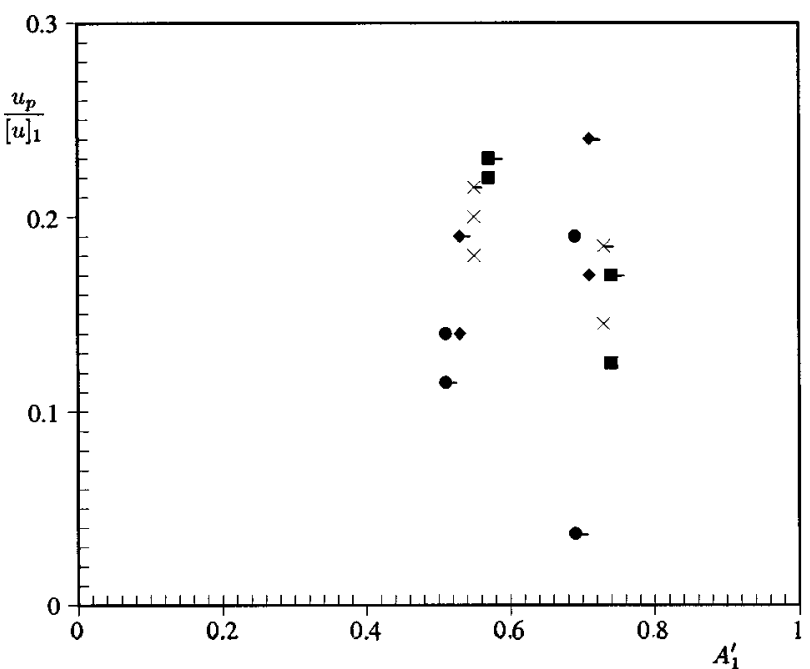

FIG. 20. Wall vortex penetration speed (nondimensional) vs reshocked Atwood ratio. Long period experiments with light/heavy interfaces. Incident shock Mach number: $1.12, \downarrow: 1.32, \times$ : $1.48, \mathbf{\square}: 1.66$. Points denoted by — refer to interfaces with large initial thicknesses, i.e., $\delta \approx 30-40 \mathrm{~mm}$ for these interfaces, as opposed to $\delta \approx 10-20 \mathrm{~mm}$ for the thinner ones. The uncertainty on $u_{p} /[u]_{1}$ is \pm 0.03 .

\section{Test gas contamination}

The mechanism for the generation of the wall vortex at an interface, namely, the Richtmyer-Meshkov instability, is different than that which causes reflected shock waves in molecular gases to bifurcate on the side walls of shock tubes. Thus, the use of a monoatomic test gas would not alleviate the wall vortex effect, as seen in Fig. 18(a) for an air/Xe interface, though the occurrence of bifurcation may aggravate it. In studies of the Richtmyer-Meshkov instability and in reflected-shock tunnels, it is necessary that the test sections be designed large enough that the wall vortices and bifurcation bubbles do not occupy the entire flow or that the long-range effects of vortex induction do not seriously compromise the accuracy of measurements.

In particular, the mechanism of wall vortex formation presented here can explain the results of Stalker and Crane ${ }^{15}$ who, for certain experimental conditions, observed test gas contamination in reflected shock tunnels even though shock bifurcation was absent.

To quantify these effects experimentally, the axial penetration of the wall vortices into the test gas was measured for long period experiments with the light/heavy continuous interfaces. Figure 20 shows the wall vortex penetration speed $u_{p}$, measured in a reference frame moving with the interface, after the interaction of the interface with the first reshock; $u_{p}$ is normalized by the interface velocity change induced by the first reshock $[u]_{1}$. This data is plotted against the "reshocked" Atwood ratio $A_{1}^{\prime} \equiv\left(\rho_{\text {test }}^{\prime}-\rho_{\text {air }}^{\prime}\right) /\left(\rho_{\text {test }}^{\prime}+\rho_{\text {air }}^{\prime}\right)$, where $\rho_{\text {air }}^{\prime}$ and $\rho_{\text {test }}^{\prime}$ designate the density of the air and test gas, respectively, after the interaction of the first reflected wave with the interface. The multiple points for a given interface at a fixed shock strength are for different interface initial thicknesses. If the value of penetration velocity measured in the laboratory frame of reference (given by $u_{p}$ $+[u]_{0}-[u]_{1}$ ), is directed toward the end of the tube (i.e., is 
positive), the wall vortex can ultimately contaminate the entire test gas slug by making its way to the end wall of the shock tube. The large scatter in the results does not allow specific conclusions to be drawn with regards to the effect of shock Mach number or interface thickness and this is due mostly to the large uncertainty in tracking the extent of the wall vortex.

\section{E. Small-scale perturbations on initially smooth interfaces}

It is also found that $2 \mathrm{D}$ and $3 \mathrm{D}$ wave fronts are generated by disturbances on the interface and on the side and end walls of the shock tube, and by interaction with the boundary layers. After these waves reverberate between the side and end walls of the shock tube and the interface itself, they can be responsible for the introduction of small perturbations on the interface, which can explain the observed thickening of initially smooth thick interfaces. ${ }^{3,4,19}$ These effects have to be kept in mind when comparing results from experiments performed in different facilities, where the geometry and experimental conditions are not the same.

\section{CONCLUSIONS}

The development of viscous boundary layers on the side walls of the shock tube in the fluid in motion behind the incident shock wave can cause the bifurcation of the reflected waves and thereafter the formation of wall bubbles and interface-contaminating jets. The appearance of these bifurcation wave patterns has been observed in the context of the Richtmyer-Meshkov instability and there were successfully compared with the models of Mark and Hess. The generation of vortical structures by shock wave-boundary layer interaction at the interface has also been demonstrated. Even if wave bifurcation arguments preclude the appearance of interface penetration jets, significant contamination can be caused by the wall vortex mechanism. The need for experimental methods to distinguish the effects of these wall vortices from the primary phenomena under study has also been pointed out. Moreover, the strain field induced by the vorticity in these wall structures tends to thin the interface; the magnitude of this effect in most of the present experiments is estimated to be of order $10 \%$ for discontinuous interfaces and $50 \%$ for continuous interfaces. The accurate characterization of interface phenomena requires the identification of extraneous effects introduced by the experimental apparatus. This could be achieved more easily by using different flow visualization and measurement techniques. The comparison of results obtained from different facilities, where the geometry and experimental conditions are not the same, is difficult to achieve, since the accurate quantification of most of these effects is not yet achievable. In general, it can be concluded that most of the side effects of the experimental investigation of the Richtmyer-Meshkov instability can be alleviated by performing experiments in large test sections at near atmospheric initial pressure.

${ }^{1}$ R. D. Richtmyer, "Taylor instability in shock acceleration of compressible fluids,” Commun. Pure Appl. Math. 8, 297 (1960).
${ }^{2}$ E. E. Meshkov, "Instability of the interface of two gases accelerated by a shock wave,'’ Sov. Fluid Dynamics 4, 101 (1969).

${ }^{3}$ M. Brouillette and B. Sturtevant, "Growth induced by multiple shock waves normally incident on plane gaseous interfaces," Physica D 37, 248 (1989).

${ }^{4}$ M. Brouillette and B. Sturtevant, "Experiments on the RichtmyerMeshkov instability: Small-scale perturbations on a plane interface," Phys. Fluids A 5, 916 (1993).

${ }^{5}$ M. Brouillette and B. Sturtevant, "Experiments on the RichtmyerMeshkov instability: Single-scale perturbations on a continuous interface," J. Fluid Mech. 263, 271 (1994).

${ }^{6} \mathrm{M}$. Brouillette, Ph.D. thesis, California Institute of Technology, 1989.

${ }^{7}$ R. Bonazza, Ph.D. thesis, California Institute of Technology, 1992.

${ }^{8}$ H. Mirels, NACA TN-3401 (1955).

${ }^{9} \mathrm{H}$. Mirels, "Shock tube test time limitation due to turbulent-wall boundary layer,', AIAA J. 2, 84 (1964).

${ }^{10} \mathrm{H}$. Mirels, "Turbulent boundary layer behind constant velocity shock including wall blowing effects," AIAA J. 22, 1042 (1983).

${ }^{11} \mathrm{H}$. Mark, "The interaction of a reflected shock wave with the boundary layer in a shock tube," J. Aeronaut. Sci. 24, 304 (1957).

${ }^{12}$ S. Byron and N. Rott, "On the interaction of the reflected shock wave with the laminar boundary layer on the shock tube walls," in Proceedings of the 1961 Heat Transfer and Fluid Mechanics Institute (Stanford University Press, Standford, California, 1961), p. 38.

${ }^{13}$ R. V. Hess, "Interaction of moving shocks and hot layers," NACA TN4002 (1957).

${ }^{14}$ L. Davies and J. L. Wilson, "Influence of reflected shock and boundarylayer interaction on shock-tube flows," Phys. Fluids 12, 37 (1969).

${ }^{15}$ R. J. Stalker and K. C. A. Crane, "Driver gas contamination in a highenthalpy reflected shock tunnel," AIAA J. 16, 277 (1978).

${ }^{16}$ W. J. Glowacki, A. L. Kuhl, H. M. Glaz, and R. E. Ferguson, "Shock wave interaction with high sound speed layer," in Shock Waves and Shock Tubes, edited by D. Bershader and R. Hanson (Stanford University Press, Stanford, 1986), p. 188.

${ }^{17}$ P. A. Thompson, Compressible-Fluid Dynamics (McGraw-Hill, New York, 1972).

${ }^{18}$ L. Houas, A. Farhat, and R. Brun, "Shock induced Rayleigh-Taylor instability in the presence of a boundary layer," Phys. Fluids 31, 807 (1988).

${ }^{19}$ G. Rodriguez, I. Galametz, H. Croso, and J.-F. Haas, "RichtmyerMeshkov instability in a vertical shock tube,' in Shock Waves@Marseille $I V$, Proceedings of the 19th International Symposium on Shock Waves, edited by R. Brun and L. Dumitrescu (Springer, New York, 1995), p. 275.

${ }^{20} \mathrm{M}$. Vetter and B. Sturtevant, "Experiments on the Richtmyer-Meshkov instability of an air/SF 6 interface," Shock Waves 4, 247 (1995).

${ }^{21} \mathrm{R}$. Bonazza and B. Sturtevant, "X-ray measurements of growth rates at a gas interface accelerated by shock waves," Phys. Fluids 8, 2496 (1996).

${ }^{22}$ J. W. Jacobs, "Shock-induced mixing of a light-gas cylinder," J. Fluid Mech. 234, 629 (1992).

${ }^{23}$ J. M. Budzinski, R. F. Benjamin, and J. W. Jacobs, "Influence of initial conditions on the flow patterns of a shock-accelerated thin fluid layer," Phys. Fluids 6, 3510 (1994).

${ }^{24}$ A. I. Abamukov, V. Yu. Fadeev, S. I. Kholkin, E. E. Meshkov, V. V. Nikiforov, P. N. Nizovtzev N. N. Sadilov, S. K. Sobolev, V. A. Tilkunov, V. O. Tochilin, A. I. Tolshmyakov, and N. V. Zhidov, "Studies of film effects on the turbulent mixing zone evolution in shock tube experiments," in Proceedings of the Fifth International Workshop on Compressible Turbulent Mixing, edited by R. Young, J. Glimm, and B. Boston (World Scientific, Singapore, 1996), p. 118.

${ }^{25}$ M. V. Bliznetsov, Y. V. Vlasov, V. I. Dudin, E. E. Meshkov, A. A. Nikulin, V. A. Til'kunov, A. I. Tolshmyakov, and S. A. Kholkin, "How the film may control the gas-gas turbulent mixing development in shock tube experiments," in Proceedings of the Sixth International Workshop on Compressible Turbulent Mixing, edited by G. Jourdan and L. Houas (Imprimerie Caractère, Marseille, 1997), p. 90.

${ }^{26}$ S. G. Zaitsev, E. V. Lazareva, V. V. Chernukha, and V. M. Belyaev, "Intensification of mixing at the interface between media of different densities upon the passage of a shock wave through it," Sov. Phys. Dokl. 30, 579 (1985).

${ }^{27} \mathrm{~L}$. Rosenhead, "The formation of vortices from a surface of discontinuity,’ Proc. R. Soc. London, Ser. A 134, 170 (1931). 